\title{
FY 1996 Cost Savings by Program
}

Date Published

April 1997

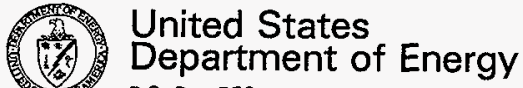

P.O. Box 550

Richland, Washington 99352 
LEGAL DISCLAMMER

This report was preparod as an account of work sponsored by an agency of the United States Government. Neither the United States Government nor any agency thereof, nor any of their employees, nor any of their contractors, subcontractors or their employees, makes any warranty, express or implied, or assumes any legal liability or responsibility for the accuracy, completeness, or any third party's use or the results of such use of any information, apparatus, product, or process disclosed, or represents that its use would not infringe privately owned rights. Reference herein to any specific commercial product, process, or service by trade name, trademark, manufacturer, or otherwise, does not necessarily constitute or imply its endorsement, recommendation, or favoring by the United States Government or any egency thereof or its contractors or subcontractors. The views and opinions of authors expressed herein do not necessarily state or reflect those of the United States Government or any agency thereof.

This report has been reproduced from the best available copy. Avallable in paper copy and microfiche.

Available to the U.S. Department of Energy and its contractors from

Office of Scientific and Technical Information

P.O. Box 62

Oak Ridge, TN 37831

(615) $576-8401$

Available to the public from the U.S. Department of Commerce National Technical Information Service

5285 Port Royal Road

Springfield, VA 22161

(703) $487-4650$

Printed in the United States of Americe

,

DISCLM-1.CHP (1-91) 
DOE/RL-97-41

\section{(EM) Baseline Scorecard System (Part 1)}

Status of Savings Reported by Major Program Report through 4th Qtr, FY 1996

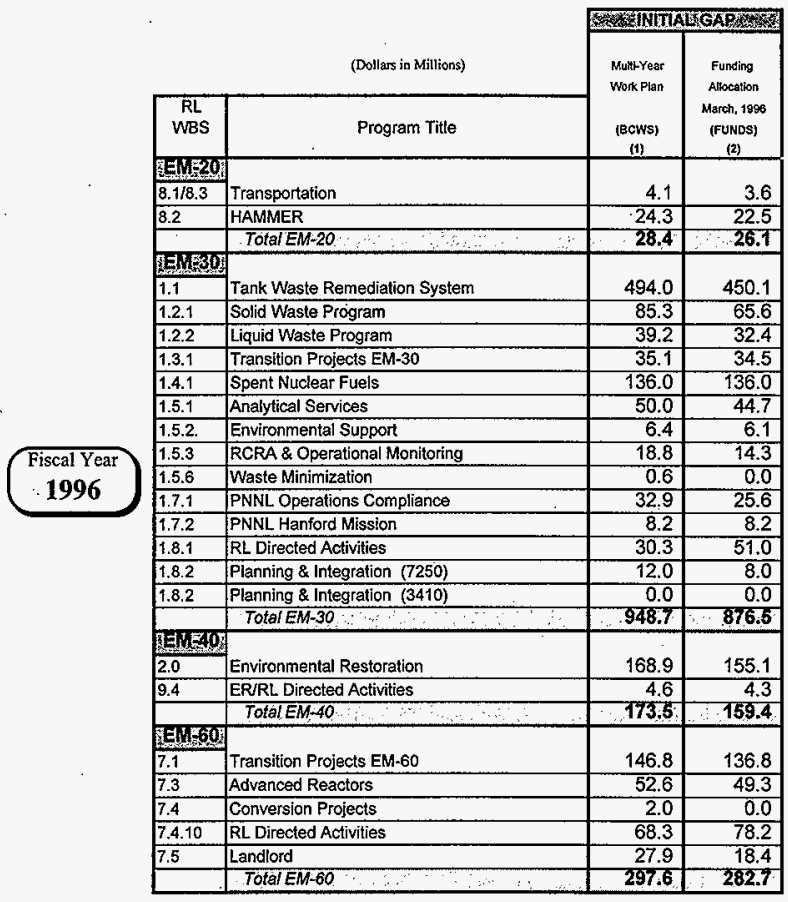

\begin{tabular}{|c|c|c|c|}
\hline \multicolumn{4}{|c|}{ OW' } \\
\hline SAVINOS & EFFICIENCY & POTENRIAL: & ADDITON'S \\
\hline $\begin{array}{l}\text { CR Approved } \\
\text { Scope } \\
\text { Deletions } \\
\text { (SAVINGS) } \\
\text { (3) }\end{array}$ & $\begin{array}{l}\text { PTS Report } \\
\text { (all Contractors) } \\
\text { (CV) } \\
\text { (4) }\end{array}$ & \begin{tabular}{c|} 
Savings \\
To \\
Date \\
$(3+4)$ \\
$(5)$
\end{tabular} & $\begin{array}{l}\text { Worksoope } \\
\text { Addations' } \\
\text { Accelerations } \\
\text { (I1+12) } \\
\text { (6) }\end{array}$ \\
\hline 0.0 & 1.5 & 1.5 & 0.5 \\
\hline 0.0 & $(0.3)$ & $(0.3)$ & 0.0 \\
\hline 0.0 & 12 & 1.2 & 0.5 \\
\hline (15.1) & 7.5 & (7.6) & 38.2 \\
\hline$(11.4)$ & (12.4) & $(23.8)$ & 5.6 \\
\hline$(5.2)$ & $(1.4)$ & $(6.6)$ & 0.7 \\
\hline 0.6 & 0.9 & 1.5 & 0.1 \\
\hline 2.5 & 0.8 & 3.3 & 7.0 \\
\hline$(4.2)$ & 0.0 & $(4.2)$ & 2.8 \\
\hline 0.0 & (3.1) & (3.1) & 0.2 \\
\hline (3.4) & 0.3 & (3.1) & 0.0 \\
\hline 0.0 & 0.0 & 0.0 & 0.3 \\
\hline (3.5) & $(0.3)$ & $(3.8)$ & 3.7 \\
\hline$(0.2)$ & 0.0 & $(0.2)$ & 0.7 \\
\hline 0.0 & 0.0 & 0.0 & 22.3 \\
\hline (2.9) & $(0.3)$ & $(3.2)$ & 0.7 \\
\hline $\begin{array}{c}0.0 \\
(42.8)\end{array}$ & $\begin{array}{c}0.0 \\
(8.0)\end{array}$ & $\begin{array}{c}0.0 \\
(50.8)\end{array}$ & $\begin{array}{r}0.0 \\
82.1\end{array}$ \\
\hline$(14.8)$ & (9.5) & (24.3) & 27.7 \\
\hline$(0.3)$ & $(0.4)$ & $(0.7)$ & 0.0 \\
\hline (15.1) & $(99)$ & $(25.0)$ & 27.7 \\
\hline (5.0) & (4.9) & $(9.9)$ & 3.5 \\
\hline 0.0 & $(1.7)$ & $(1.7)$ & 6.0 \\
\hline 0.0 & 0.0 & 0.0 & 0.0 \\
\hline 0.0 & 0.0 & 0.0 & 4.3 \\
\hline$(10.2)$ & 0.0 & $(10.2)$ & 4.1 \\
\hline$(16,3)$ & $(6.6)$ & (21.9) & $17: 9$ \\
\hline
\end{tabular}

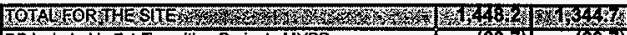
\begin{tabular}{|l|l|l|}
\hline DP included in 7.1 Transition Projects MYPP & (20.7) & (20.7) \\
\hline
\end{tabular}

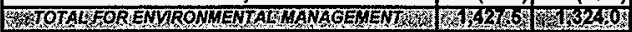

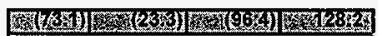

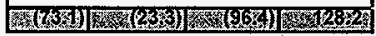


(EM) Baseline Scorecard System (Part 1)

Status of Savings Reported by Major Program

Report through 4th Qtr, FY 1996

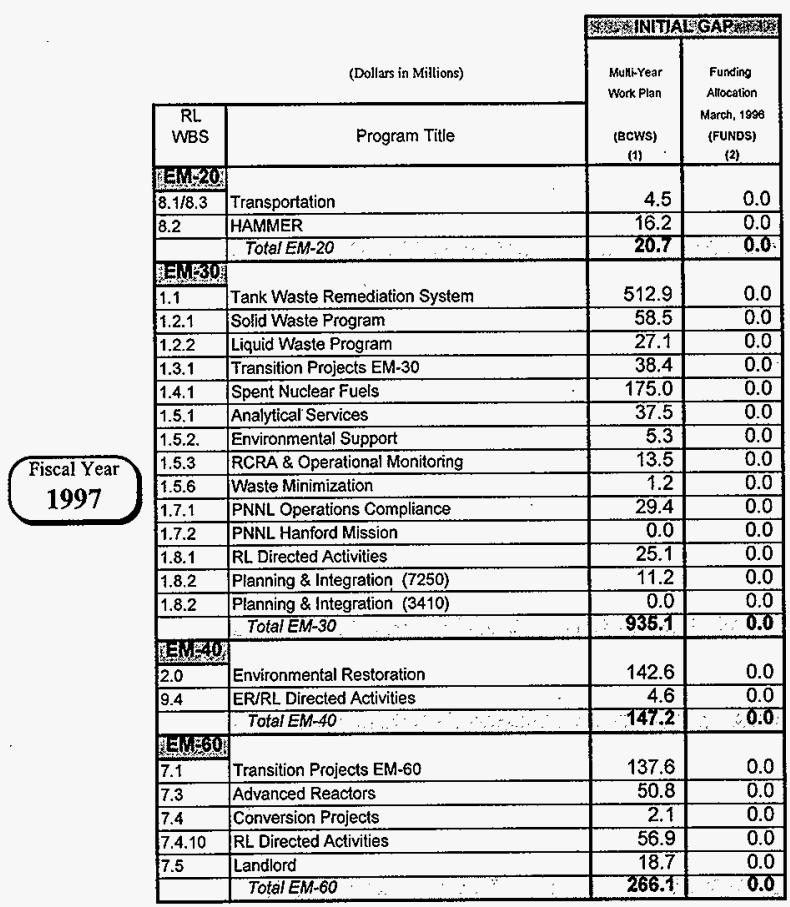

\begin{tabular}{|c|c|c|c|}
\hline \multicolumn{4}{|c|}{ 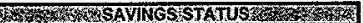 } \\
\hline SAVINGS & EFICLENCY. & POTENTIAL: & ADomons: \\
\hline $\begin{array}{l}\text { CR Approved } \\
\text { Scopo } \\
\text { Deletions } \\
\text { (SAVINGS) } \\
\text { (3) }\end{array}$ & $\begin{array}{l}\text { PIS Report } \\
\text { (all Conlractors) } \\
\text { (cV) } \\
\text { (4) }\end{array}$ & $\begin{array}{l}\text { Savings } \\
\text { To } \\
\text { Date } \\
(3+4) \\
(5)\end{array}$ & $\begin{array}{l}\text { Workscope } \\
\text { Adsitionsi } \\
\text { Accelerations } \\
(11+12) \\
\text { (6) }\end{array}$ \\
\hline 0.0 & & 0.0 & 0.0 \\
\hline 0.0 & & 0.0 & 0.0 \\
\hline 0.0 & 0.0 & 0.0 & 0,0 \\
\hline$(19.0)$ & & $(19.0)$ & 5.4 \\
\hline 0.0 & & 0.0 & 3.3 \\
\hline 0.0 & & 0.0 & $(0.2)$ \\
\hline$(1.7)$ & & $(1.7)$ & $(0.1)$ \\
\hline (6.9) & & $(6.9)$ & 17.8 \\
\hline (1.8) & & $(1.8)$ & 0.0 \\
\hline 0.0 & & 0.0 & 0.0 \\
\hline 0.0 & & 0.0 & 0.0 \\
\hline 0.0 & & 0.0 & 0.0 \\
\hline 0.0 & & 0.0 & $(0.0)$ \\
\hline 0.0 & & 0.0 & 0.6 \\
\hline 0.0 & & 0.0 & 0.0 \\
\hline 0.0 & & 0.0 & 0.0 \\
\hline 0.0 & & 0.0 & 0.0 \\
\hline (29.4) & 0.0 & $(29.4)$ & 26.8 \\
\hline (1.8) & & (1.8) & 1.4 \\
\hline 0.0 & & 0.0 & 0.0 \\
\hline (7.8) & 0.0 & $(1.8)$ & 7.4 \\
\hline (2.3) & & (2.3) & 0.3 \\
\hline 0.0 & & 0.0 & (19) \\
\hline 0.0 & & 0.0 & 0.0 \\
\hline 0.0 & & 0.0 & 0.0 \\
\hline$(0.7)$ & & $(0.7)$ & $(0.5)$ \\
\hline$(3.0)$ & 0.0 & $(3.0)$ & $(2,2)$ \\
\hline
\end{tabular}

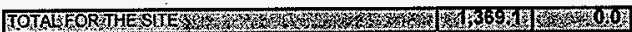

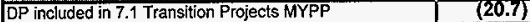

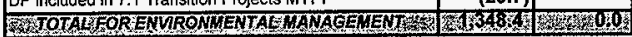

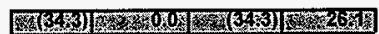

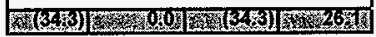


(EM) Baseline Scorecard System (Part 1)

Status of Savings Reported by Major Program

Report through 4th Qtr, FY 1996

\begin{tabular}{|c|c|c|c|c|}
\hline & & \multirow[b]{2}{*}{ (Dollars in Millions) } & \multicolumn{2}{|c|}{ 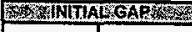 } \\
\hline & . & & \multirow{2}{*}{$\begin{array}{c}\text { Mutsyear } \\
\text { Work Pisn } \\
\text { (Baws) } \\
\text { (1) }\end{array}$} & \multirow{2}{*}{$\begin{array}{c}\text { Funcing } \\
\text { Allocation } \\
\text { March, 1995 } \\
\text { (Fundos) } \\
\text { (2) } \\
\end{array}$} \\
\hline & $\begin{array}{c}\text { RL } \\
\text { WES }\end{array}$ & Program Title & & \\
\hline & Yentezo & & & \\
\hline & $8.1 / 8.3$ & Transportation & 4.9 & 0.0 \\
\hline & 8.2 & HAMMER & 4.9 & 0.0 \\
\hline & & Total EM-20 & 9.8 & 0.0 \\
\hline & $\mathrm{BHSO3}$ & & & \\
\hline & 1.1 & Tank Waste Remediation System & 495.7 & 0.0 \\
\hline & 1.2 .1 & Solid Waste Program & 51.2 & 0.0 \\
\hline & 1.2 .2 & Liquid Waste Program & 28.0 & 0.0 \\
\hline & $\overline{1.3 .1}$ & Transition Projects EM-30 & $40 . \overline{0}$ & 0.0 \\
\hline & 1.4 .1 & Spent Nuclear Fuels & 123.0 & 0.0 \\
\hline & 1.5 .1 & Analytical Services & 35.7 & 0.0 \\
\hline & 1.5 .2 . & Environmental Support & 4.6 & 0.0 \\
\hline Fiscal Year & 1.5 .3 & RCRA \& Operational Monitoring & 12.8 & 0.0 \\
\hline 1008 & 1.5 .6 & Waste Minimization & 1.0 & 0.0 \\
\hline & 1.7 .1 & PNNL Operations Compliance & 25.4 & 0.0 \\
\hline & 1.7 .2 & PNNL Hanford Mission & $0 . \overline{0}$ & 0.0 \\
\hline & 1.8.1 & RL Directed Activities & 29.8 & 0.0 \\
\hline & 1.8 .2 & Planning \& Integration (7250) & 9.2 & 0.0 \\
\hline & 1.8 .2 & Planning \& integration (3410) & $0 . \overline{0}$ & 0.0 \\
\hline & & Totel EM-30 & 856.4 & 0.0 \\
\hline & gET40 & & & \\
\hline & 2.0 & Environmental Restoration & 94.6 & 0.0 \\
\hline & 9.4 & ER/RL Directed Activities & 4.6 & 0.0 \\
\hline & & Total EM 40 & 99.2 & 0.0 \\
\hline & ES150 & & & \\
\hline & 7.1 & Transition Projects EM-60 & 114.9 & 0.0 \\
\hline & 7.3 & Advanced Reactors & 46.6 & 0.0 \\
\hline & 7.4 & Conversion Projects & 2.1 & 0.0 \\
\hline & 7.4 .10 & RL Directed Activities & 62.0 & 0.0 \\
\hline & 7.5 & Landlord & 13.1 & 0.0 \\
\hline & & Total EM-60 & 238.7 & 0.0 \\
\hline
\end{tabular}

\begin{tabular}{|c|c|c|c|}
\hline \multicolumn{4}{|c|}{ mys } \\
\hline SAVINGS & EFFICIENCY & POTENMAL: & ADDITIONS \\
\hline $\begin{array}{l}\text { CR Approved } \\
\text { Scopt } \\
\text { Defelions } \\
\text { (SAVINGS) } \\
\text { (3) }\end{array}$ & $\begin{array}{l}\text { PIS Ricport } \\
\text { (all Contractors) } \\
\text { (cV) } \\
\text { (4) }\end{array}$ & $\begin{array}{l}\text { Sawhos } \\
\text { To } \\
\text { Date } \\
\text { (3+4) } \\
\text { (5) }\end{array}$ & $\begin{array}{c}\text { Workscopo } \\
\text { Adtritionst } \\
\text { Accelerations } \\
(11+12) \\
(6)\end{array}$ \\
\hline 0.0 & & 0.0 & 0.0 \\
\hline 0.0 & & 0.0 & 0.0 \\
\hline 0.0 & 0.0 & 0.0 & 0.0 \\
\hline (21.6) & & (21.6) & 5.3 \\
\hline 0.0 & & 0.0 & 0.0 \\
\hline 0.0 & & $\overline{0.0}$ & 0.0 \\
\hline (3.1) & & $(3.1)$ & 0.0 \\
\hline$(13.0)$ & & $(13.0)$ & 9.6 \\
\hline (1.2) & & $(1.2)$ & 0.0 \\
\hline 0.0 & & 0.0 & 0.0 \\
\hline 0.0 & & 0.0 & 0.0 \\
\hline 0.0 & & 0.0 & 0.0 \\
\hline 0.0 & & 0.0 & 0.0 \\
\hline 0.0 & & 0.0 & 0.0 \\
\hline 0.0 & & 0.0 & 0.0 \\
\hline 0.0 & & 0.0 & 0.0 \\
\hline 0.0 & & 0.0 & 0.0 \\
\hline$(38.9)$ & 0.0 & (38.9) & 14.9 \\
\hline$(0.0)$ & & $(0.0)$ & 11.0 \\
\hline 0.0 & & 0.0 & 0.0 \\
\hline$(0.0)$ & 0.0 & $(0.0)$ & 170 \\
\hline (3.8) & & (3.8) & 0.0 \\
\hline 0.0 & & 0.0 & $(2.0)$ \\
\hline 0.0 & & 0.0 & 0.0 \\
\hline 0.0 & & 0.0 & 0.0 \\
\hline 0.0 & & 0.0 & 0.0 \\
\hline$(3.8)$ & 0.0 & $(3.8)$ & $(2.0)$ \\
\hline
\end{tabular}

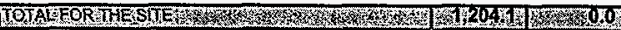

\begin{tabular}{|l|r|r|}
\hline$D P$ included in 7.1 Transition Projects MYPP & $(20.7)$ & 0.0
\end{tabular}

3.

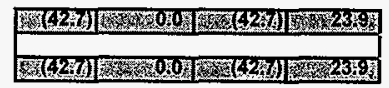


DOERL-97-41

Note: PROGRAM TITLE: The WBS breakout on the scorecard will not reflect the new EM-70 structure until FY 1997.

Note: COLUMN (1): 10/20/95 Multi-Year Work Plan (BCWS)

Note: COLUMN (2): This is the funding allocation received in March, 1997 from HQ

Note: COLUMN (3): CR Approved Scope Deletions (SAVINGS). This column comes directly from (COLUMN 8) Part 2, Baseline Scorecard System.

Note: COLUMN (4): May be adjusted per program input. 
(EM) Baseline Scorecard System (Part 2)

Status of Baseline and Funding Changes Reported by Major Program

Report through 4th Qtr, FY 1996

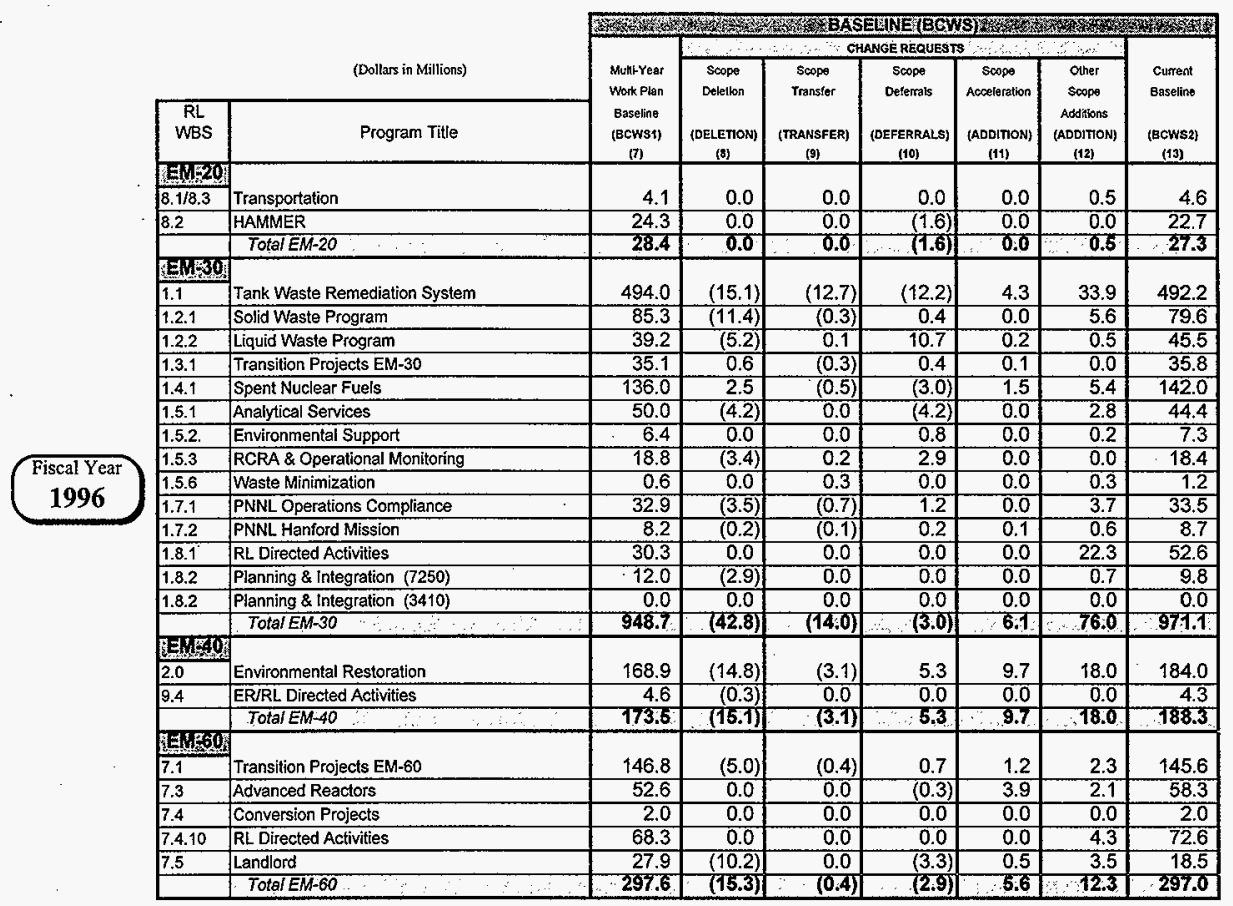

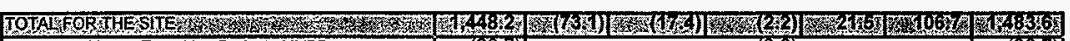
\begin{tabular}{|l|r|r|r|}
\hline DP included in 7.1 Transition Projects MYPP & $(20.7)$ & $(2.0)$ & (22.7)
\end{tabular}

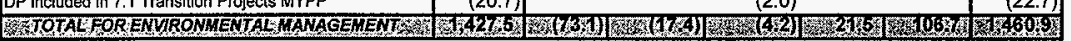


(EM) Baseline Scorecard System (Part 2)

Status of Baseline and Funding Changes Reported by Major Program Report through 4th Qtr, FY 1996

\begin{tabular}{|c|c|c|c|c|c|c|c|c|c|}
\hline & \multirow{3}{*}{\multicolumn{2}{|c|}{ (Dollers in Millions) }} & \multicolumn{7}{|c|}{ 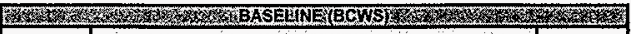 } \\
\hline & & & \multirow{3}{*}{$\begin{array}{l}\text { Munityear } \\
\text { mork Plan } \\
\text { Basetina } \\
\text { (Bcws) } \\
\text { (f) }\end{array}$} & \multicolumn{5}{|c|}{ CHANGEREQUESTS } & \multirow{3}{*}{$\begin{array}{c}\text { Current } \\
\text { Easeline } \\
\text { (BCWs) } \\
\text { (13) }\end{array}$} \\
\hline & & & & \multirow{2}{*}{$\begin{array}{c}\text { Scoppe } \\
\text { Delelion } \\
\text { (DELLETOSN) } \\
\text { (B) }\end{array}$} & \multirow{2}{*}{$\begin{array}{c}\text { Soope } \\
\text { Transfer } \\
\text { (TRANSFER) } \\
\text { (9) }\end{array}$} & \multirow{2}{*}{\begin{tabular}{|c|} 
Scope \\
Defertals \\
\\
(DEFERRALS) \\
(10) \\
\end{tabular}} & \multirow{2}{*}{$\begin{array}{c}\text { Scopse } \\
\text { Accoserefation } \\
\cdot \\
\text { (ADOTION) } \\
\text { (11) }\end{array}$} & \multirow{2}{*}{$\begin{array}{c}\text { Other } \\
\text { scopos } \\
\text { Aderitions } \\
\text { (ADDTrTom) } \\
\text { (12) }\end{array}$} & \\
\hline & $\begin{array}{l}\mathrm{RL} \\
\text { WBS }\end{array}$ & Program Title & & & & & & & \\
\hline & 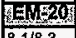 & & & & & & & & \\
\hline & $8.1 / 8.3$ & Transportation & 4.5 & 0.0 & 0.0 & 0.0 & 0.0 & 0.0 & 4.5 \\
\hline & 8.2 & HAMMER & 16.2 & 0.0 & 0.0 & 0.0 & 0.0 & 0.0 & 16.2 \\
\hline & & Total EM-20 & 20.7 & 0.0 & 0.0 & 0.0 & 0.0 & 0.0 & 20.7 \\
\hline & EDU.30 & & & & & & & & \\
\hline & 1.1 & Tank Waste Remediation System & 512.9 & (19.0) & (139.7) & 13.1 & 0.6 & 4.8 & 372.7 \\
\hline & 1.2 .1 & Solid Waste Program & 58.5 & 0.0 & 0.0 & 3.0 & 0.0 & 3.3 & 64.8 \\
\hline & 1.2 .2 & Liquid Waste Program & 27.1 & 0.0 & 0.0 & 3.0 & $(0.2)$ & 0.0 & 29.9 \\
\hline & 1.3 .1 & Transition Projects EM-30 & 38.4 & (1.7) & 0.0 & 0.2 & $(0.1)$ & 0.0 & 36.8 \\
\hline & 1.4.1 & Spent Nuclear Fuels & 175.0 & $(6.9)$ & 2.3 & 0.2 & 1.0 & 16.9 & 188.5 \\
\hline v & 1.5 .1 & Analytical Services & 37.5 & $(1.8)$ & 0.0 & 4.5 & 0.0 & 0.0 & 40.2 \\
\hline & 1.5 .2$. & Environmental Support & 5.3 & 0.0 & 0.0 & 0.0 & 0.0 & 0.0 & 5.3 \\
\hline Fiscal Year & 1.5 .3 & RCRA \& Operational Monitoring & 13.5 & 0.0 & 0.0 & 2.3 & 0.0 & 0.0 & 15.8 \\
\hline Motati that & 1.5 .6 & Waste Minimization & 1.2 & 0.0 & 0.0 & 0.0 & 0.0 & 0.0 & 1.2 \\
\hline 1997 & 1.7 .1 & PNNL Operations Compliance & 29.4 & 0.0 & 7.0 & 0.0 & $(0.0)$ & 0.0 & 36.4 \\
\hline & 1.7 .2 & PNNL Hanford Mission & 0.0 & 0.0 & 0.0 & 0.1 & 0.0 & 0.6 & 0.6 \\
\hline & 1.8 .1 & RL Directed Activities & 25.1 & 0.0 & 0.0 & 0.0 & 0.0 & 0.0 & 25.1 \\
\hline & 1.8 .2 & Planning \& Integration (7250) & 11.2 & 0.0 & 0.0 & 0.0 & 0.0 & 0.0 & 11.2 \\
\hline & 1.8 .2 & Planning \& Integration (3410) & 0.0 & 0.0 & 0.0 & 0.0 & 0.0 & 0.0 & 0.0 \\
\hline & & Total EM-30 & 935.1 & $(29.4)$ & $(13003)$ & 26.4 & 7.2 & 25.6 & 828.6 \\
\hline & EEMEO & & & & & & & & \\
\hline & 2.0 & Environmental Restoration & 142.6 & (1.8) & 0.0 & 10.4 & 0.0 & 1.4 & 152.6 \\
\hline & 9.4 & ER/RL Directed Activities & 4.6 & 0.0 & 0.0 & 0.0 & 0.0 & 0.0 & 4.6 \\
\hline & & Total EM-40 & 747.2 & $(1,8)$ & 0.0 & 10.4 & 0.0 & 1.4 & 157.2 \\
\hline & DEMEO & & & & & & & & \\
\hline & 7.1 & Transition Projects EM-60 & 137.6 & (2.3) & 2.3 & 1.8 & 0.0 & 0.3 & 139.7 \\
\hline & 7.3 & Advanced Reactors & 50.8 & 0.0 & 0.0 & 0.3 & $(1.9)$ & 0.0 & 49.2 \\
\hline & $7 . \overline{4}$ & Conversion Projects & $2: 1$ & 0.0 & 0.0 & 0.0 & 0.0 & 0.0 & 2.1 \\
\hline & 7.4 .10 & RL Directed Activities & 56.9 & 0.0 & 0.0 & 0.0 & 0.0 & 0.0 & 56.9 \\
\hline & 7.5 & Landlord & 18.7 & $(0.7)$ & 0.0 & 3.0 & $(0.5)$ & 0.0 & 20.5 \\
\hline & & Total EM-60 & 266.1 & $(3.0)$ & 2.3 & 5.1 & $(2.4)$ & 0,3 & 268.4 \\
\hline & TORALE & ORTHESITE T & 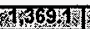 & 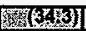 & 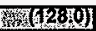 & 250 & 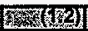 & & \\
\hline & DP includ & Ied in 7.1 Transition Projects MYPP & $(20.7)$ & & & & & & $(20.7)$ \\
\hline & 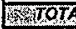 & GLFORENVRONMENTALMANAE & 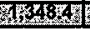 & 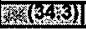 & 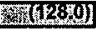 & TW & (3) & 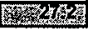 & 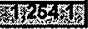 \\
\hline
\end{tabular}


(EM) Baseline Scorecard System (Part 2)

Status of Baseline and Funding Changes Reported by Major Program Report through 4th Qtr, FY 1996

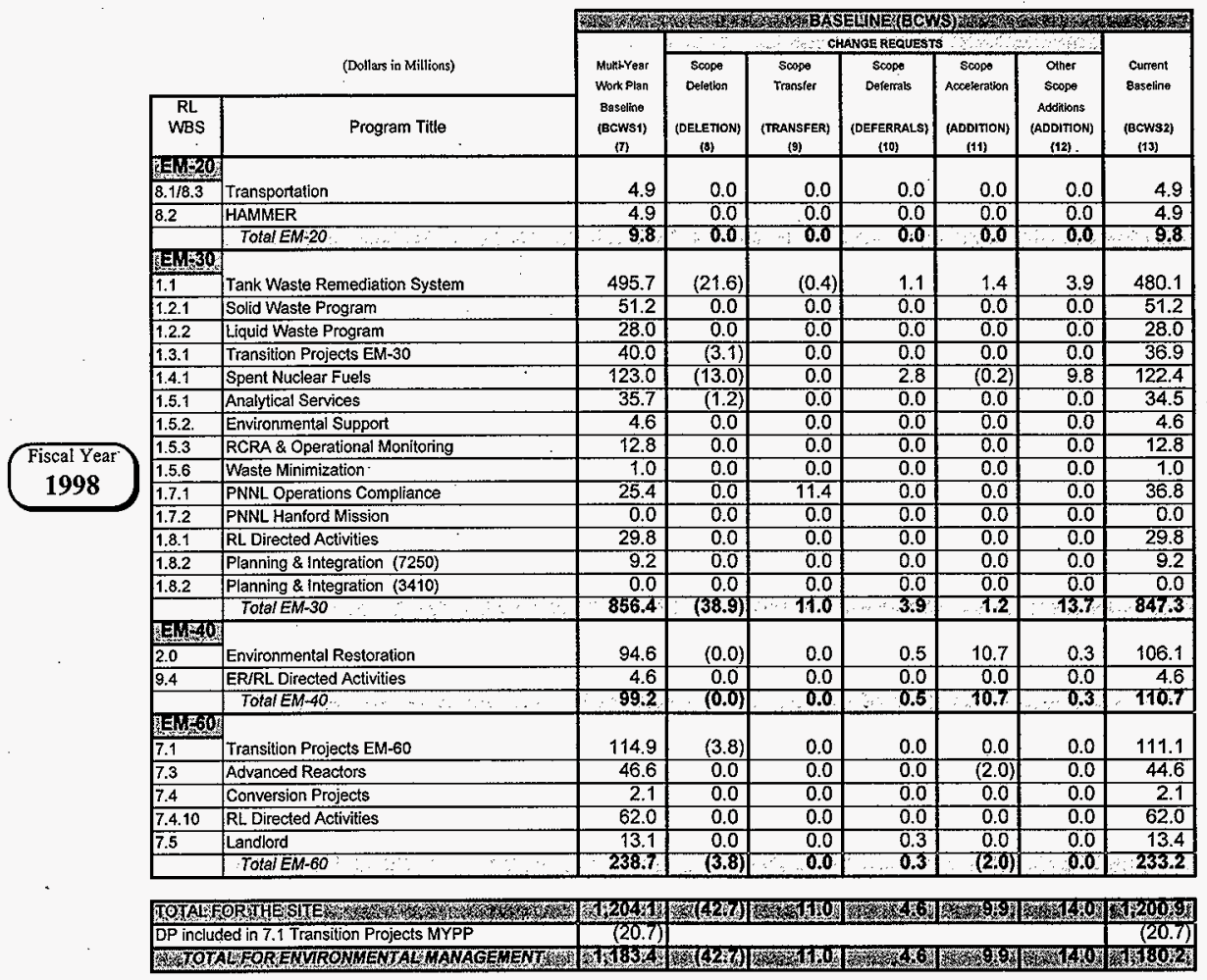


Note: PROGRAM TITLE: The WBS breakout on the scorecard will not reflect the new EM-70 structure until FY 1997.

Note: COLUMN (7): 10/20/95 Multi-Year Work Plan (8CWS1) or equivalent baseline (i.e. RL Directed). This is the basis for measuring all workscope performance and savings

Note: COLUMN (8): Scope Deletion (DELETION). All change request amounts that represent work scope reductions, deletions, process step reductions and deletions. Does not include scope deferrals.

Note: COLUMN (9): Scope Transfer (TRANSFER). All change request amounts that represent work scope transfers. DOES NOT COUNT TOWARDS SAVINGS.

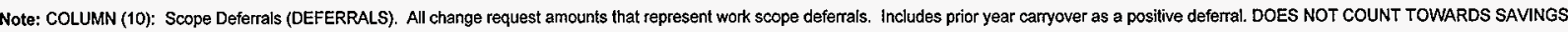

Note: COLUMN (11): Scope Acceleration (ADDITIONS). All change request amounts that represent work scope accelerations.

Note: COLUMN (12): Other Scope Additions (ADOITIONS). All change request amounts that represent other work scope additions.

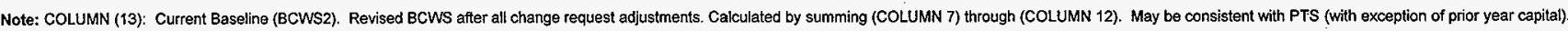


(EM) Baseline Scorecard System (Part 2)

Program Detail

\subsection{HAMMER}

\begin{tabular}{|c|c|c|c|c|c|c|c|c|}
\hline \multirow[b]{2}{*}{$\begin{array}{l}\text { WBs } \\
\text { Program } \\
\text { Elotront }\end{array}$} & \multirow[b]{2}{*}{ CR No. } & & \multicolumn{6}{|c|}{ FY 19\& BASELINE SCOPE CHANGES } \\
\hline & & $\begin{array}{l}\text { Change Request } \\
\text { Treteronsectigtion }\end{array}$ & $\begin{array}{c}\text { (EM) } \\
\text { Scose } \\
\text { Detition }\end{array}$ & $\begin{array}{c}\text { (SM) } \\
\text { Seopes } \\
\text { Transfor }\end{array}$ & $\begin{array}{c}\text { (BM) } \\
\text { Scope } \\
\text { Doforrals }\end{array}$ & $\begin{array}{c}\text { (SWM } \\
\text { Scops } \\
\text { Accolesetion }\end{array}$ & $\begin{array}{c}\text { (AH) } \\
\text { Scopos } \\
\text { Addtltons }\end{array}$ & $\begin{array}{l}\text { (5:M) } \\
1936 \\
\text { Total }\end{array}$ \\
\hline & & PTS Adjustment & & & $(1.60)$ & & & $(1.60)$ \\
\hline & & & & & & & & 0.00 \\
\hline & & & & & & & & 0.00 \\
\hline & & & & & & & & 0.00 \\
\hline & & & & & & & & 0.00 \\
\hline TOTAL & & & 0.00 & 0.00 & $(1.60)$ & 0.00 & 0.00 & $(1.60)$ \\
\hline
\end{tabular}

\begin{tabular}{|c|c|c|c|c|c|}
\hline \multicolumn{6}{|c|}{ FY TSOZ BASELANE SCOPE CHANGES } \\
\hline $\begin{array}{l}\text { SSM } \\
\text { Scope } \\
\text { Dotiotion }\end{array}$ & $\begin{array}{c}\text { (sth) } \\
\text { Scopes } \\
\text { Transfor }\end{array}$ & $\begin{array}{c}\text { (SH) } \\
\text { Scope } \\
\text { Deforrats }\end{array}$ & $\begin{array}{c}\text { (SM) } \\
\text { scose } \\
\text { Accoloration }\end{array}$ & $\begin{array}{c}\text { (sM) } \\
\text { scope } \\
\text { Additions }\end{array}$ & $\begin{array}{l}\text { (5N) } \\
1997 \\
\text { Totat }\end{array}$ \\
\hline & & & & & 0.00 \\
\hline & & & & & 0.00 \\
\hline & & & & & 0.00 \\
\hline & & & & & 0.00 \\
\hline & & & & & 0.00 \\
\hline 0.00 & 0.00 & 0.00 & 0.00 & 0.00 & 0.00 \\
\hline
\end{tabular}

\begin{tabular}{|c|c|c|c|c|c|c|}
\hline \multicolumn{6}{|c|}{ FY 1098 B̈ASELINE SCOPE CHANGES } & \multirow[b]{2}{*}{$\begin{array}{c}\text { (SM) } \\
\text { CR } \\
\text { TOTAL }\end{array}$} \\
\hline $\begin{array}{c}\text { (SM) } \\
\text { Scopes } \\
\text { Desectlon }\end{array}$ & $\begin{array}{c}\text { (sM) } \\
\text { Scope } \\
\text { Transter }\end{array}$ & $\begin{array}{c}\text { (SM) } \\
\text { Scopes } \\
\text { Dethraly }\end{array}$ & $\begin{array}{c}\text { (5M) } \\
\text { Scope } \\
\text { Accelorentlon }\end{array}$ & $\begin{array}{c}\text { (SM) } \\
\text { Scope } \\
\text { Additions }\end{array}$ & $\begin{array}{l}\text { (SM) } \\
\text { 19ss } \\
\text { Total }\end{array}$ & \\
\hline & & & & & 0.00 & $(1.60)$ \\
\hline & & & & & 0.00 & 0.00 \\
\hline & & & & & 0.00 & 0.00 \\
\hline & & & & & 0.00 & 0.00 \\
\hline & & & & & 0.00 & 0.00 \\
\hline 0.00 & 0.00 & 0.00 & 0.00 & 0.00 & 0.00 & $(1.60)$ \\
\hline
\end{tabular}



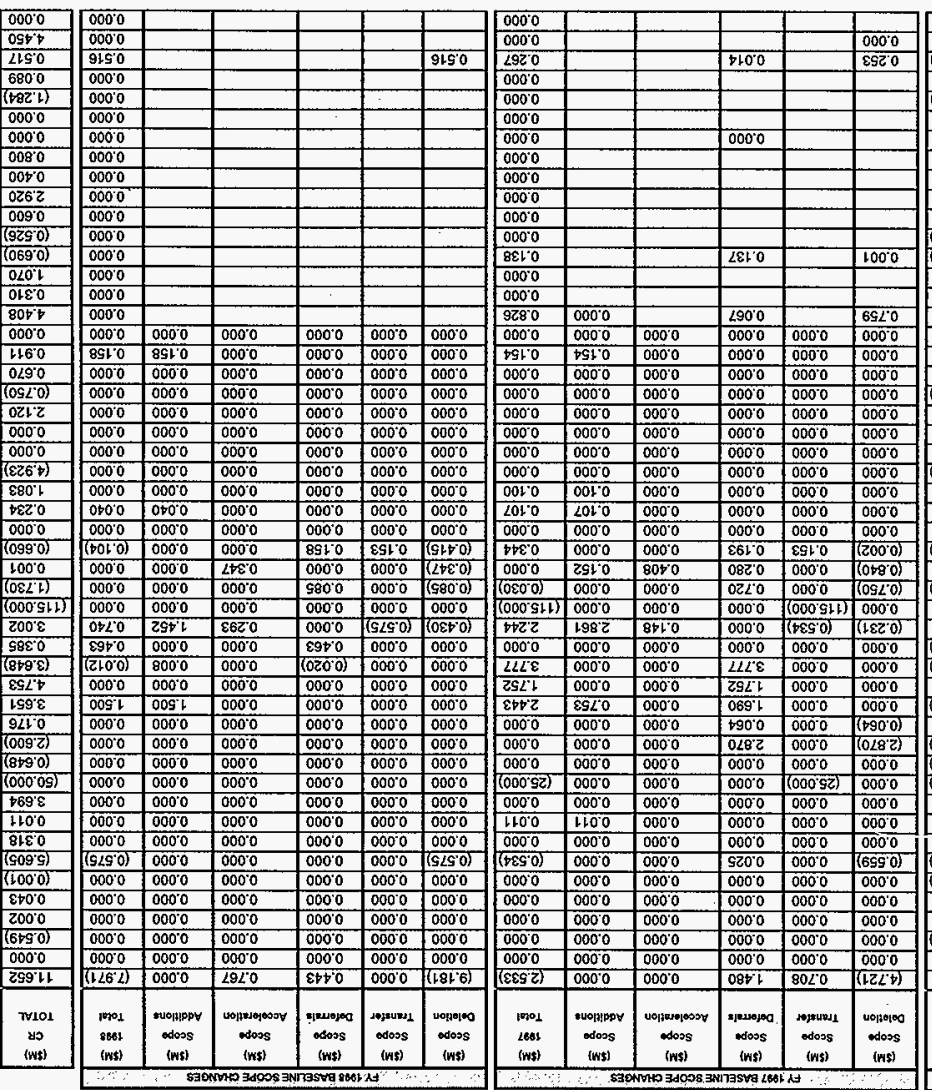

\begin{tabular}{|c|c|c|c|c|c|c|c|c|}
\hline $000 \%$ & 0920 & 0000 & 0000 & 0000 & $(\cos 20)$ & & $900-96-2 \mathrm{din}$ & \\
\hline ost & PlZO & 0000 & 0000 & 0000 & 9EZ 7 & & 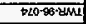 & \\
\hline$(9920)$ & $000^{\circ} 0$ & $000^{\circ} 0$ & (p100) & $000^{\circ} 0$ & $\left(z s z^{2} 0\right)$ & & $20006 \mathrm{rms}$ & \\
\hline $680^{\circ} 0$ & $\angle E b^{\circ} 0$ & $69+0$ & $\frac{\left.168^{\circ} 0\right)}{(6)}$ & $000^{\circ} 0$ & $\frac{100^{\circ} 0}{10}$ & & $820-60-8 \mathrm{dm}$ & \\
\hline$\left(+\$ 8 z^{\prime} L\right)$ & 0000 & $000^{\prime} 0$ & 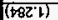 & $000^{\circ} 0$ & $000^{\circ} 0$ & & $920.06 .8 \mathrm{kMI}$ & \\
\hline 0000 & 78000 & $000^{\circ}$ & $000 \%$ & 0000 & $\left(180^{\circ} 0\right)$ & & $220-00 \mathrm{sec}$ & \\
\hline $000^{\circ}$ & $000^{\circ} 0$ & $000^{\circ}$ & $000^{\circ} 0$ & $000^{\prime} 0$ & 0000 & & $180 \cdot 02.8 \mathrm{dM}$ & \\
\hline $008^{\circ} 0$ & $008^{\circ} 0$ & $000^{\circ} 0$ & $000^{\circ} 0$ & $000^{\circ} 0$ & 0000 & & $600 \cdot 0606 \mathrm{sin}$ & \\
\hline 0060 & $000^{\circ} 0$ & $000^{\circ} 0$ & 0000 & 0000 & 0000 & & $200-66-2 \mathrm{dm}$ & \\
\hline $026 \mathrm{Z}$ & 0012 & $000^{\circ} 0$ & 0000 & $028^{\prime} \mathrm{L}$ & 0000 & & $020-28 \times d \mathrm{dMI}$ & \\
\hline 0090 & 0090 & 0000 & 0000 & 0000 & 0000 & & $600 \cdot 96 \cdot \mathrm{din}$ & \\
\hline$(9290)$ & $00 z^{\circ} 0$ & $000 \%$ & 0.000 & 0000 & $(92 \angle 0)$ & & $590-88-4 \mathrm{ML}$ & \\
\hline$(8280)$ & $000^{\circ} 0$ & 0000 & (Z\&10) & 0000 & $\left(969^{\circ}\right)$ & & 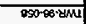 & \\
\hline $0 \angle 0^{\circ} \mathrm{l}$ & $0 \angle 0^{\prime} t$ & $000 \%$ & $0000^{\circ}$ & $000^{\circ} 0$ & $000^{\circ} 0$ & & 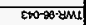 & \\
\hline 0180 & 0180 & $000 \%$ & $000^{\circ} 0$ & $000^{\circ} 0$ & 0000 & & 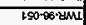 & \\
\hline$z 8^{\prime} \varepsilon$ & $000^{\prime} 0$ & $000^{\prime} 0$ & $\left(290^{\circ} 0\right)$ & $000^{\circ} 0$ & $659^{\circ} \varepsilon$ & & $230-80-\mathrm{dml}$ & \\
\hline $000^{\circ}$ & $89 L^{\circ} 0$ & $000^{\prime} 0$ & $000^{\circ} 0$ & $000^{\prime} 0$ & $\frac{185.0)}{(89)}$ & & $250 \cdot 28-2 \mathrm{em}$ & Fin \\
\hline $669^{\circ} 0$ & $000^{\circ} 0$ & $000^{\prime} 0$ & $000^{\circ} 0$ & $6+9^{\prime} 0$ & $090^{\circ} 0$ & & 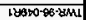 & tit \\
\hline $0 \angle 9^{\prime} a$ & $0 \angle 9^{\circ} 0$ & $000^{\prime} 0$ & 0000 & $000^{\circ} 0$ & $000^{\circ} 0$ & & 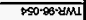 & WT \\
\hline$(0 \log 20)$ & $000^{\circ}$ & $000^{\circ} 0$ & 0000 & $000^{\circ} 0$ & $\left(\cos \lambda^{\prime} 0\right)$ & & $+00-20-\sin 1$ & Bith \\
\hline $0 z k^{2}$ & 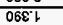 & $021 \%$ & (89s'0) & $000^{\circ} 0$ & $\frac{1}{(281 \cdot 0)}$ & & 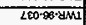 & 17 \\
\hline $000 \%$ & 0000 & $000 \%$ & 0000 & 0000 & 0000 & & $510-58-y, y=$ & $1 \pi$ \\
\hline 0000 & 0000 & 0000 & $000^{\circ} 0$ & $000^{\circ}$ & 0000 & & $6 \cos ^{2}-88^{\circ} \mathrm{dMA}$ & $5 \pi$ \\
\hline$\left(\varepsilon 2 \sigma^{\prime} t\right)$ & $000^{\circ} 0$ & $000^{\circ} 0$ & $000^{\circ} 0$ & $\left(\varepsilon 26^{\prime} b\right)$ & 0000 & & $500-80-8 \mathrm{MMI}$ & $\sqrt{1+3}$ \\
\hline $866^{\circ} 0$ & $\varepsilon 86^{\circ} 0$ & $000 \%$ & $000^{\circ} 0$ & $000^{\circ} 0$ & 0000 & & $050-28-d \mathrm{dM}$ & 178 \\
\hline $280^{\circ}$ & 2710 & $000^{\circ} 0$ & $000^{\prime} 0$ & $000^{\circ} 0$ & $\left(980^{\circ}\right)$ & & $860-88-4 \times 1 \times 1$ & 173 \\
\hline $000^{\circ} 0$ & $980^{\circ} 0$ & $000^{\circ} 0$ & $000^{\circ} 0$ & $000^{\circ} 0$ & (980 & & $s+0-80-4 \mathrm{MN}$ & 513 \\
\hline$\left(006^{\circ} \circ\right)$ & $000^{\circ} 0$ & 0000 & $\left(685^{\circ}\right)$ & $000^{\circ} 0$ & (LเEO) & & 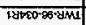 & 174 \\
\hline 6000 & 8510 & stso & $\left(80 \varepsilon^{\circ}\right)$ & $000^{\circ} 0$ & (\$680) & & 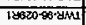 & 19 \\
\hline$(00 \angle L)$ & $000^{\circ} 0$ & 0000 & $\left(508^{\circ} 0\right)$ & $000^{\circ}$ & $\left(968^{\circ}\right)$ & & 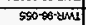 & $i \pi$ \\
\hline 0000 & $000^{\circ} 0$ & $000^{\circ} 0$ & $000^{\circ} 0$ & $000^{\circ} 0$ & $000^{\circ} 0$ & & 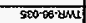 & $1 \%$ \\
\hline 8100 & $000^{\circ}$ & 7901 & $000^{\circ} 0$ & 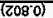 & $\left(z \not z^{\prime} 0\right)$ & & $6+0-28-8 \mathrm{~m}$ & 134 \\
\hline$\left(820^{\circ} 0\right)$ & ost't & $000^{\circ} 0$ & (zzद⿺辶) & $000^{\circ}$ & $\left(9+\mathbf{Z}^{\prime \prime \prime}\right)$ & 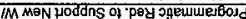 & 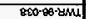 & \\
\hline$\left(\varepsilon\left\llcorner b^{*} L\right)\right.$ & $98 \varepsilon^{\prime} \varepsilon$ & $020^{\circ} \mathrm{t}$ & $(024.8)$ & $0+L L^{\prime} 0$ & $\left.(89)^{\prime} \varepsilon\right)$ & 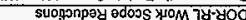 & 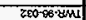 & 17. \\
\hline $1000^{\circ} \varepsilon$ & 9l6!t & 0000 & (द⿳⺈口: & 0008 & (89.०) & 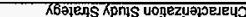 & 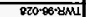 & Tht \\
\hline$(26 \mathrm{Z} 0)$ & 8009 & 0000 & $(009$ \%) & $000^{\circ}$ & $(002 t)$ & 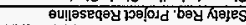 & $\$ 20-98 \cdot-8 \mathrm{ML}$ & Ith \\
\hline $9 \angle 20$ & $0+20$ & 0000 & $\left(\operatorname{tg} 90^{\circ} 0\right)$ & 0000 & 0000 & 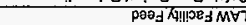 & 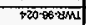 & हाT \\
\hline$(009 z)$ & 0000 & 0000 & $(0092)$ & 0000 & 0000 & 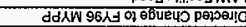 & $270 \cdot 03 \cdot 8 \mathrm{ML}$ & 171 \\
\hline$(8+9-0)$ & $988 \mathrm{l}$ & 0000 & $000^{\circ} 0$ & 0000 & (1.Esz) & 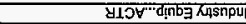 & 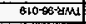 & Tit \\
\hline$(000 \mathrm{og})$ & 0000 & 0000 & $000^{\circ} 0$ & $\left(000^{\prime}, 52\right)$ & $000^{\circ} 0$ & uonjanpey jasping uonezgnenyd & sio-so: & $\pi 3$ \\
\hline $669^{\circ} \varepsilon$ & 0000 & 0000 & $\not 69^{\circ} \varepsilon$ & 0000 & 0000 & JLMW-Fo' & $910 \cdot 38 \cdot \mathrm{dMI}$ & tin \\
\hline $000^{\circ} 0$ & $+80^{\circ}$ & 0000 & $800^{\circ} 0$ & $\left(20^{\circ} 0\right)$ & $000 \%$ & Euldo & $210-90-\mathrm{EMN}$ & $i n$ \\
\hline $8 I \varepsilon 0$ & $106^{\prime} 9$ & 0000 & 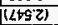 & $000 \%$ & $\left(980^{\circ}, 8\right)$ & 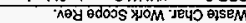 & $110-5 \times 3 \times 1$ & in \\
\hline$(96+7)$ & Doz'o & 0000 & $\left(s 20^{\circ}\right)$ & $(000$ ' & $\left(L 299^{2}\right)$ & 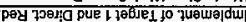 & 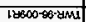 & तit \\
\hline$(1,000)$ & $000^{\circ}$ & 0000 & 00000 & 0000 & $(1000)$ & 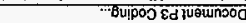 & 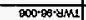 & 17 \\
\hline 8500 & 0000 & 0000 & 00000 & $\varepsilon p 0^{\prime} 0$ & 0000 & 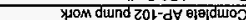 & copo- $08-4 \mathrm{ym}$ & Fiteith \\
\hline 2000 & ost't & $000^{\circ} 0$ & 0000 & 2000 & (Oosin) & 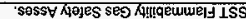 & 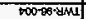 & int \\
\hline$\left(16+5^{\circ} 0\right)$ & $000^{\circ}$ & 0000 & $000^{\circ} 0$ & $\frac{\left(6 \circ g^{\prime} 0\right)}{(0)}$ & 0000 & 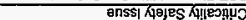 & $8600-58-8 \times 4=$ & bis \\
\hline & 0000 & $000^{\prime} 0$ & 0000 & $000^{\prime} 0$ & 0000 & $(26 \lambda-\exists)$ )noesol0 pue uofinjosay SIE & ह00:58:dMn| & तit \\
\hline 99122 & $000^{\circ} 0^{\circ}$ & 0000 & $98 \% " 9$ & $060^{9} 91$ & $089^{\circ} 0$ & eul|gsequy & $100-58 \mathrm{am}$ & $\cos ^{2} 1$ \\
\hline poss & $\begin{array}{l}\text { suopluppy } \\
\text { cocoss }\end{array}$ & 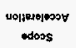 & Now19000 & ofominas & $\begin{array}{l}\text { Hegyyos } \\
\text { sodoss }\end{array}$ & 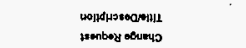 & 口NบO & 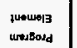 \\
\hline (H3) & (א\$) & \{Ks: & (सs) & (ws) & (พ\$s) & & & $\operatorname{sen}$ \\
\hline
\end{tabular}

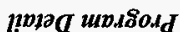

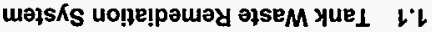

( 


\begin{tabular}{|c|c|c|c|c|c|c|c|c|c|c|c|c|}
\hline$\left(989^{\prime}\right.$ 'Gl) & $\left(089^{\prime} 51\right)$ & $898^{\prime} \varepsilon$ & $\angle 00^{\prime} \mid$ & $621 \%$ & $\left(Z Z b^{\circ} 0\right)$ & $\left(200^{\prime}, 2\right)$ & $(\angle D Z C D L)$ & $88^{\circ 7}$ & $9 S S^{\circ} 0$ & $690^{\circ} \varepsilon 1$ & $\left(\varepsilon \angle 9^{\circ} 6 \in L\right)$ & $\left(\angle 80^{\circ} 61\right)$ \\
\hline 2091 & 0000 & & & & & & $000^{\circ} 0$ & & & & & \\
\hline$\left(860^{\circ} \mathrm{L}\right)$ & $\left(980^{\circ} L 1\right)$ & & & & & $\left(980^{\circ} i 1\right)$ & $(\varepsilon 1001)$ & & & & & $\left(\varepsilon 0^{\prime} 01\right)$ \\
\hline 0000 & $000^{\circ} 0$ & & & & & & $000^{\circ} 0$ & & & & & \\
\hline 0952 & $00 \angle 0$ & $00 \angle 0$ & & & & & $00 \angle 0$ & $00 \angle 0$ & & & & \\
\hline 0000 & \begin{tabular}{|l|l}
$000^{\circ} 0$ \\
\end{tabular} & & & & & & 0000 & & & & & \\
\hline $\begin{array}{l}\text { TYLOOL } \\
\text { U5 } \\
\text { (W5) }\end{array}$ & $\begin{array}{l}\text { inos } \\
\text { Pret } \\
\text { (H)s }\end{array}$ & $\begin{array}{l}\text { suo|yppy } \\
\text { odoss } \\
\text { (Wf) }\end{array}$ & $\begin{array}{c}\text { vopesopeory } \\
\text { cotoss } \\
\text { (ws) }\end{array}$ & $\begin{array}{l}\text { s;enejed } \\
\text { edors } \\
\text { (ms) }\end{array}$ & $\begin{array}{l}\text { Aoprouss } \\
\text { odooss } \\
\text { (ws) }\end{array}$ & $\begin{array}{l}\text { Loflema } \\
\text { odors } \\
\text { (ms) }\end{array}$ & $\begin{array}{l}1 \mathrm{~mol} \\
\text { isst } \\
\text { (ws) }\end{array}$ & $\begin{array}{l}\text { olopppy } \\
\text { edess } \\
\text { (ws) }\end{array}$ & 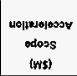 & $\begin{array}{c}\text { andopos } \\
\text { adoss } \\
\text { (W\$) }\end{array}$ & 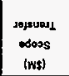 & $\begin{array}{l}\text { wopespoc } \\
\text { odoss } \\
\text { (WF) }\end{array}$ \\
\hline
\end{tabular}

\begin{tabular}{|c|c|c|c|c|c|c|c|c|}
\hline$\left(608^{\circ}\right)$ & SO6. $E$ E & L9ED & 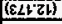 & (20<-20) & (860's) & & & $7 \forall 101$ \\
\hline 2091 & $\angle L B^{\prime} \mathrm{L}$ & 0000 & $000^{\circ} 0$ & 0000 & $\left(0, Z^{\circ} 0\right)$ & & $800-80-4 \mathrm{ML}$ & 131 \\
\hline $000^{\circ} 0$ & $000^{\circ} 0$ & $000^{\circ} 0$ & $000^{\circ} 0$ & 0000 & $000^{\circ} 0$ & & $250.568 \mathrm{HML}$ & \\
\hline $000^{\circ} 0$ & OSL 0 & $000^{\circ} 0$ & $000^{\circ} 0$ & 0000 & $(0 \log )$ & & $580-80-8 \mathrm{MI}$ & t' \\
\hline $09 \angle 0$ & $09 L^{\prime} 0$ & $000^{\circ} 0$ & $000^{\circ} 0$ & $000^{\circ} 0$ & $000^{\prime} 0$ & & $120-86-14 \mathrm{ML}$ & in \\
\hline $000^{\circ} 0$ & $000^{\circ} 0$ & $000^{\circ} 0$ & $000^{\circ} 0$ & 0000 & $000^{\circ} 0$ & & $500-80-8 \mathrm{dML}$ & in \\
\hline $\begin{array}{l}\text { 10901 } \\
9681 \\
\text { (พ\$?) }\end{array}$ & 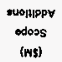 & 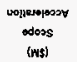 & 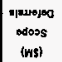 & $\begin{array}{l}\text { 10peses } \\
\text { ocoss } \\
\text { (Ws) }\end{array}$ & $\begin{array}{l}\text { uopopod } \\
\text { coloss } \\
\text { (W:5) }\end{array}$ & 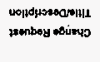 & "อN צכ & 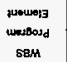 \\
\hline
\end{tabular}

\section{1mpa tums8odd}

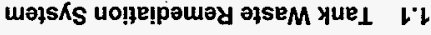

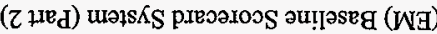

15-26-78/900 


\begin{tabular}{|c|c|c|c|c|c|c|}
\hline $829^{\circ} 0$ & $000^{\circ} 0$ & $000^{\circ} 0$ & $000^{\circ} 0$ & 0000 & 0000 & $000^{\circ} 0$ \\
\hline 0000 & 0000 & & & & & \\
\hline $000^{\circ} 0$ & 0000 & & & & & \\
\hline$(2600)$ & 0000 & & & & & \\
\hline 0000 & 0000 & & & & & \\
\hline $000^{\circ} 0$ & $000^{\circ} 0$ & & & & & \\
\hline $890^{\circ} 0$ & 0000 & & & & & \\
\hline 091.0 & $000^{\prime} 0$ & & & & & \\
\hline$(8800)$ & $000^{\circ} 0$ & & & & & \\
\hline $000^{\circ} 0$ & $000^{\circ} 0$ & & & & & \\
\hline (211.0) & 0000 & & & & & \\
\hline 0000 & $000^{\circ} 0$ & & & & & \\
\hline $9 \angle 6^{\prime} t$ & $000^{\prime} 0$ & & & & & \\
\hline 0000 & $000^{\prime} 0$ & & & & & \\
\hline 0000 & $000^{\prime} 0$ & & & & & \\
\hline $092^{\prime \prime}$ & 0000 & & & & & \\
\hline$\left(996^{\circ} 01\right)$ & 0000 & & & & & \\
\hline $000^{\circ} 0$ & $000^{\prime} 0$ & & & & & \\
\hline $000^{\circ}$ & $000 \%$ & & & & & \\
\hline 0000 & $000^{\circ} 0$ & & & & & \\
\hline pol. 0 & 0000 & & & & & \\
\hline $2 \varepsilon 89$ & $000^{\circ}$ & & & & $\approx$ & \\
\hline $\begin{array}{c}\text { T101 } \\
\text { yo } \\
\text { (wst) }\end{array}$ & $\begin{array}{l}1701 \\
\text { 1066s } \\
\text { (4M3) }\end{array}$ & $\begin{array}{l}\text { suopppy } \\
\text { Nows } \\
\text { (wss) }\end{array}$ & 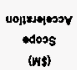 & $\begin{array}{l}\text { sousenge } \\
\text { ocoss } \\
\text { (vis) }\end{array}$ & 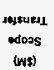 & $\begin{array}{l}\text { Lopene0 } \\
\text { ocoos } \\
\text { (Wts) }\end{array}$ \\
\hline & & & भ2.30005 & 7) & & \\
\hline
\end{tabular}

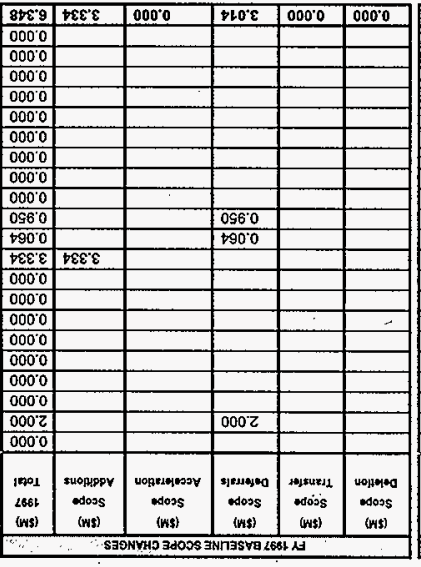

\begin{tabular}{|c|c|c|c|c|c|c|c|c|}
\hline$\left(0 z L^{\circ} s\right)$ & $289 ' s$ & 0000 & $8 \angle \varepsilon^{\circ} 0$ & $\left(06 z^{\circ} 0\right)$ & $\left(\mathrm{G} 6 \mathrm{E}^{\prime} \mathrm{t}\right)$ & & & 7V1OL \\
\hline \multicolumn{9}{|l|}{$000^{\circ} 0$} \\
\hline $000^{\prime} 0$ & & & & & & & & \\
\hline$(260.0)$ & 0000 & 0000 & $000^{\prime} 0$ & $\left(\angle 60^{\prime} 0\right)$ & $000^{\circ} 0$ & & $120-96-\mathrm{ans}$ & \\
\hline $000^{\prime} 0$ & 0200 & $000^{\circ} 0$ & $000^{\prime} 0$ & $000^{\prime} 0$ & $\left(020^{\circ} 0\right)$ & & 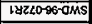 & \\
\hline $000^{\prime} 0$ & Ollo & 0000 & $000^{\prime} 0$ & (01!O). & $000^{\prime} 0$ & & $180-96-0 \mathrm{MS}$ & \\
\hline $850^{\circ} 0$ & $850^{\circ} 0$ & 0000 & 0000 & $000^{\prime} 0$ & $000^{\circ} 0$ & & $080-56-0 \mathrm{MS}$ & \\
\hline 0910 & $091 \%$ & 0000 & $000 \%$ & $000^{\circ} 0$ & $000^{\circ} 0$ & & 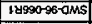 & \\
\hline$(8800)$ & $000^{\circ} 0$ & 0000 & $000^{\circ} 0$ & $\left.880^{\circ} 0\right)$ & $000^{\circ} 0$ & & 850-96-0.MS & \\
\hline 0000 & 0120 & 0000 & 0000 & 0000 & $\left(012^{\prime} 0\right)$ & & 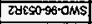 & \\
\hline$(2901)$ & $000^{\circ} 0$ & 0000 & $(0560)$ & $000^{\circ}$ & (나우) & & 190-96-9MS & \\
\hline$(+900)$ & $000^{\circ} 0$ & 0000 & $\left(\$ 90^{\circ} 0\right)$ & $000^{\circ} 0$ & 0000 & & 290-96-OMS & \\
\hline 279.1 & $269 \%$ & $000^{\circ} 0$ & $000^{\prime} 0$ & $000^{\prime} 0$ & $000^{\circ} 0$ & & $\angle 50-96-0 M S$ & \\
\hline 0000 & $000^{\circ} 0$ & 0000 & 0000 & $000^{\circ} 0$ & $000^{\circ} 0$ & & 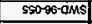 & $|2|$ \\
\hline 0000 & $8 \angle 0 Z$ & 0000 & $000^{\circ} 0$ & 0000 & $(820 \mathrm{Z})$ & & $\varepsilon \varepsilon 0-96 \% \mathrm{MS}$ & 12 \\
\hline 0920 & 0920 & 0000 & 0000 & 0000 & 0000 & & sro-96-aMS & 27 \\
\hline (\$960) & 0000 & 0000 & $(\mathrm{~s} 50 \mathrm{Z})$ & 0000 & $(0268)$ & & sc0-960 MS & I2I \\
\hline 0000 & $000^{\circ} 0$ & 0000 & $000^{\circ} 0$ & 0000 & 0,000 & & $110-98 \mathrm{ams}$ & I6i \\
\hline $000^{\circ} 0$ & $000^{\circ} 0$ & 0000 & 0000 & $000 \%$ & 0000 & & $010-96-0 \mathrm{Ms}$ & 126 \\
\hline 0000 & 8290 & $000^{\circ} 0$ & $\left(829^{\prime} 0\right)$ & $000^{\circ} 0$ & $000^{\circ} 0$ & & $800-96-0 \mathrm{MS}$ & 126 \\
\hline (968') & $b \angle t 0$ & 0000 & $(\angle 9 E Z)$ & 0000 & 0000 & & $000-96-0 \mathrm{MS}$ & 126 \\
\hline 2889 & & & $2 \varepsilon \varepsilon 9$ & & & 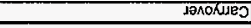 & ONS6) & 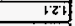 \\
\hline $\begin{array}{l}1001 \\
9661 \\
\text { (W5) }\end{array}$ & $\begin{array}{l}\text { suopeppy } \\
\text { odoss } \\
\text { (w\$) }\end{array}$ & $\begin{array}{c}\text { uoptropasor } \\
\text { edoos } \\
\text { (kss) }\end{array}$ & $\begin{array}{l}\text { stenaned } \\
\text { ofors } \\
\text { (inst) }\end{array}$ & $\begin{array}{l}\text { Mysues } \\
\text { edors } \\
\text { (nts) }\end{array}$ & $\begin{array}{c}\text { uopmen } \\
\text { nosss } \\
\text { (ins) }\end{array}$ & 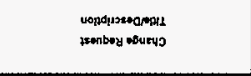 & ONYO & 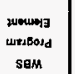 \\
\hline
\end{tabular}

\section{1"vpaQ}

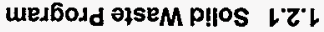

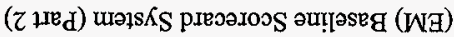

15. $26 \cdot 74 / 500$ 


\begin{tabular}{|c|c|c|c|c|c|c|}
\hline 9st.6 & $000^{\circ} 0$ & $000^{\circ} 0$ & $000^{\circ} 0$ & $000^{\circ} 0$ & $000^{\prime} 0$ & $000^{\prime} 0$ \\
\hline $000^{\prime} 0$ & 0000 & & & & & \\
\hline $000^{\circ} 0$ & $000^{\circ} 0$ & & & & & \\
\hline $000^{\circ} 0$ & $000^{\circ} 0$ & & & & & \\
\hline$\left(009^{\circ}\right)$ & $000^{\circ} 0$ & & & & & \\
\hline $999^{\circ} 01$ & $000^{\circ} 0$ & & & & & \\
\hline $\begin{array}{c}\text { w101 } \\
\text { yo } \\
\text { (ns) }\end{array}$ & $\begin{array}{l}1001 \\
0661 \\
\text { (ns) }\end{array}$ & $\begin{array}{l}\text { suopppy } \\
\text { edooss } \\
\text { (HSt) }\end{array}$ & 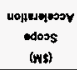 & 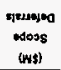 & $\begin{array}{l}\text { Aastust } \\
\text { odoos } \\
\text { (WN5) }\end{array}$ & $\begin{array}{l}\text { Wopiened } \\
\text { odors } \\
\text { (wip) }\end{array}$ \\
\hline
\end{tabular}

\begin{tabular}{|c|c|c|c|c|c|}
\hline 6P8'Z & $000^{\circ} 0$ & {$\left[\left(1 \angle t^{\prime} 0\right)\right.$} & OZO'E & $000^{\circ} 0$ & $000^{\circ} 0$ \\
\hline \multicolumn{6}{|l|}{$000^{\circ} 0$} \\
\hline $000^{\circ} 0$ & & & & & \\
\hline 0020 & & & $00 \angle 0$ & & \\
\hline$(1, \angle 20)$ & & $(1 \angle L O)$ & & & \\
\hline $0 Z \varepsilon^{\prime} Z$ & & & $0 z \varepsilon z$ & & \\
\hline $\begin{array}{l}1701 \\
2651 \\
\text { (N5) }\end{array}$ & $\begin{array}{l}\text { suomppy } \\
\text {-doss } \\
\text { (พy) }\end{array}$ & 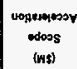 & 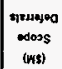 & 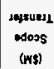 & $\begin{array}{l}\text { uopered } \\
\text { odoss } \\
\text { (WH) }\end{array}$ \\
\hline
\end{tabular}

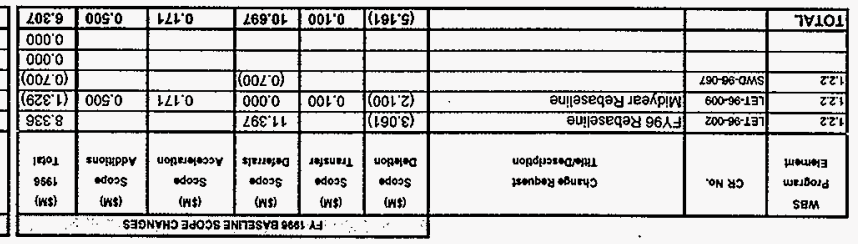

11p1aa umaso.dd

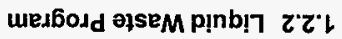

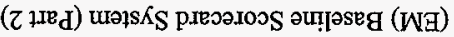

เซ-16-ามะด0 


\begin{tabular}{|c|c|c|c|c|c|c|}
\hline (SEG & (S9O'E) & $000^{\circ} 0$ & $000^{\circ} 0$ & $000^{\prime} 0$ & $000^{\circ} 0$ & $\left(590^{\circ} \varepsilon\right)$ \\
\hline $000^{\circ} 0$ & 0000 & & & & & \\
\hline 0000 & 0000 & & & & & \\
\hline$(9600)$ & $000^{\circ} 0$ & & & & & \\
\hline 0000 & $000^{\prime} 0$ & & & & & \\
\hline$\angle 960$ & $000^{\circ} 0$ & & & & & \\
\hline (घ) & $000^{\prime} 0$ & & & & & \\
\hline $000^{\circ} 0$ & $000^{\circ} 0$ & & & & & \\
\hline$\left(\varepsilon \in l^{\prime} 0\right)$ & $000^{\circ} 0$ & & & & & \\
\hline $000^{\circ} 0$ & 0000 & & & & & \\
\hline ( $\left(98 L^{\prime} g\right)$ & (G90\%) & & & & & $\left(990^{\circ} \mathrm{C}\right)$ \\
\hline $968^{\circ} 0$ & 0000 & & & & & \\
\hline $\begin{array}{c}7 \mathbf{W} 101 \\
\text { do } \\
\text { (W\$s) }\end{array}$ & $\begin{array}{l}1701 \\
9661 \\
\text { (w5) }\end{array}$ & $\begin{array}{l}\text { suophppy } \\
\text { odoss } \\
\text { (w:s) }\end{array}$ & 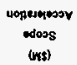 & 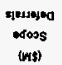 & 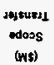 & $\begin{array}{l}\text { wopmed } \\
\text { ocoss } \\
\text { (HS) }\end{array}$ \\
\hline & $\overline{5}$ & & 4030008 & syanss & & \\
\hline
\end{tabular}

\begin{tabular}{|c|c|c|c|c|c|}
\hline (86s'L) & $000^{\circ} 0$ & $\left(960^{\circ} 0\right)$ & 8120 & $000^{\circ} 0$ & $(8+22)$ \\
\hline \multicolumn{6}{|l|}{$000^{\prime} 0$} \\
\hline \multicolumn{6}{|l|}{$000^{\prime} 0$} \\
\hline$(2100)$ & & $\left(960^{\circ} 0\right)$ & $6 \angle 0^{\prime} 0$ & & \\
\hline$(\angle L I O)$ & & & $(\angle L I 0)$ & & \\
\hline $28 \varepsilon^{\circ} 0$ & & & & & $28 \varepsilon^{\circ} 0$ \\
\hline \multicolumn{6}{|l|}{$000^{\prime} 0$} \\
\hline \multicolumn{6}{|l|}{0000} \\
\hline$(2500)$ & & & $960^{\circ} 0$ & & $\left(\varepsilon+l^{\prime} 0\right)$ \\
\hline $0 Z 20$ & & & $0 z Z^{\prime} 0$ & & \\
\hline$\left(\angle \mathrm{SB} \mathrm{C}^{\prime}\right)$ & & & & & (LSGL) \\
\hline \multicolumn{6}{|l|}{$000^{\circ} 0$} \\
\hline $\begin{array}{l}1019 \\
2661 \\
\text { (HA) }\end{array}$ & $\begin{array}{l}\text { stopsppy } \\
\text { cooss } \\
\text { (Ws) }\end{array}$ & $\begin{array}{l}\text { uspentwossy } \\
\text { wess } \\
\text { (Wf) }\end{array}$ & 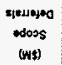 & $\begin{array}{l}\text { Aostuted } \\
\text { odoss } \\
\text { (w:) }\end{array}$ & $\begin{array}{l}\text { Lopmer } \\
\text { Nioss } \\
\text { (NS) }\end{array}$ \\
\hline & & 7970028 & $795 \times 82$ & तs & \\
\hline
\end{tabular}

\begin{tabular}{|c|c|c|c|c|c|c|c|c|}
\hline $9 z 20$ & 5600 & 9600 & $t 9 \varepsilon^{\prime} 0$ & $\left(\varepsilon\left\llcorner\varepsilon^{\prime} 0\right)\right.$ & SLS'0 & & & $7 \forall 101$ \\
\hline \multicolumn{9}{|l|}{0000} \\
\hline 0000 & & & & & & & & \\
\hline$(6 \angle 00)$ & & 9600 & (GLl0) & & & & $270-96 \cdot \mathrm{d} 1$ & \\
\hline $2 \angle 10$ & & & $\angle I L O$ & & & & 650-96-d1 & \\
\hline SLSO & & & & & GLSO & & $8 \varepsilon 0-56 \cdot d 1$ & \\
\hline (દடع & & & & $(\varepsilon \mid \bar{\varepsilon} 0)$ & & & $9 E 0-90-d 1$ & \\
\hline 0000 & & & & & & parouddess!̣ & $080096 \cdot d t$ & \\
\hline$\left(960^{\circ} 0\right)$ & & & $\left(960^{\circ} 0\right)$ & & & & $\angle E 00-96-d 1$ & \\
\hline$(0 z 20)$ & & & $(0 Z Z 0)$ & & & & $18006 . d 1$ & \\
\hline$(8910)$ & $710^{\circ} 0$ & 0000 & (LLIO) & & $000^{\circ} 0$ & & $910-96-d 1$ & $5 \mathrm{Et}$ \\
\hline St8'0 & & & St80 & & & JAAOKUEO S66L IS ISMQUEld & $900-96 \% d 1$ & iाti \\
\hline $\begin{array}{l}\text { Inol } \\
965 k \\
\text { (Ks) }\end{array}$ & $\begin{array}{l}\text { 2uopppy } \\
\text { odooss } \\
\text { (ws) }\end{array}$ & 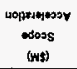 & $\begin{array}{l}\text { spenoged } \\
\text { odoss } \\
\text { (Ws) }\end{array}$ & $\begin{array}{l}\text { rogrues! } \\
\text { odors } \\
\text { (ws) }\end{array}$ & $\begin{array}{l}\text { uoplemea } \\
\text { edoss } \\
\text { (irs) }\end{array}$ & 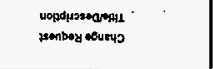 & כים & $\begin{array}{l}\text { Howng } \\
\text { wersosdd } \\
\text { s9m }\end{array}$ \\
\hline
\end{tabular}

\section{npzaq ubdsodd}

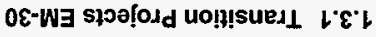

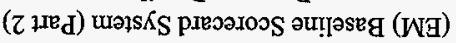

เพ-46-78ม 


\begin{tabular}{|c|c|c|c|c|c|c|}
\hline $868^{\prime 21}$ & $\left(009^{\circ} 0\right)$ & $008^{\prime} 6$ & $\left(16 \mathrm{~K}^{\circ} 0\right)$ & $8 B L 2$ & $000^{\circ} 0$ & (LEB' $Z \mathrm{Zl})$ \\
\hline $000^{\circ} 01$ & $002 t$ & $00 \varepsilon S$ & & & & $\left(009^{\circ}\right)$ \\
\hline $0 \varepsilon \varepsilon Z$ & 0000 & & & & & \\
\hline$(06+2)$ & $(0+1 z)$ & & & & & $(0+1 \bar{z})$ \\
\hline 0000 & 0000 & & & & & $000^{\circ} 0$ \\
\hline SOC' $\varepsilon$ & $\left(\not 16^{\circ} 0\right)$ & & & & & $\left(\right.$ (5) $\left.66^{\circ} 0\right)$ \\
\hline$\left(\angle 8 B^{\circ}\right)$ & $\left(760^{\circ} 1\right)$ & & & 8980 & & $\left(29 \theta^{\circ} \mathrm{L}\right)$ \\
\hline 0000 & $000^{\circ}$ & & & & & $000^{\circ} 0$ \\
\hline $00 \varepsilon^{\prime} b$ & 0000 & & & & & $000^{\circ} 0$ \\
\hline$(+21 i b)$ & $\left(\$ \angle L L^{\circ}\right)$ & & & & & $\left(\not L L^{2}\right)$ \\
\hline 0000 & 0000 & & & & & \\
\hline $000^{\circ} 0$ & $000^{\circ} 0$ & & & & & \\
\hline 0000 & 000 & & & & & \\
\hline 0000 & 0000 & & & & & \\
\hline $768^{\circ}$ & $\overline{0000}$ & & & & & \\
\hline $000^{\circ} 0$ & 0000 & & & & & \\
\hline $000^{\circ} 0$ & $000^{\circ}$ & & & & & \\
\hline 9200 & $220^{\circ} 0$ & $00 g^{\prime} \phi$ & $\left(16 L^{\circ}\right)$ & $0 \& 61$ & & (ㄴ)Z'9) \\
\hline $000^{\prime} 0$ & $000^{\circ} 0$ & & & & & \\
\hline $000^{\circ} 0$ & 000 & & & & & \\
\hline $268^{\circ} \mathrm{L}$ & $000^{\prime \prime} 0$ & & & & & \\
\hline 8080 & $000^{\circ} 0$ & & & & & \\
\hline $\begin{array}{l}\text { Tvios } \\
\text { yo } \\
\text { (ms) }\end{array}$ & $\begin{array}{l}1001 \\
2601 \\
(1+5)\end{array}$ & $\begin{array}{l}\text { suopppy } \\
\text { edoss } \\
\text { (wss) }\end{array}$ & 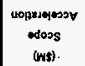 & 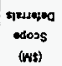 & $\begin{array}{l}\text { 10ysump } \\
\text { odors } \\
\text { (W:) }\end{array}$ & $\begin{array}{l}\text { यоpнed } \\
\text { odoss } \\
\text { (ws) }\end{array}$ \\
\hline & & 5 & 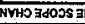 & 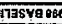 & & \\
\hline
\end{tabular}

\begin{tabular}{|c|c|c|c|c|c|}
\hline$\#$ & E98'9L & $996^{\prime} 0$ & $8+20$ & DE\&'Z & $\left(z 68^{\circ} 9\right)$ \\
\hline $008^{\circ} \mathrm{s}$ & $00 \operatorname{s}^{\prime} 6$ & & & & $\left(00 L^{\prime} \varepsilon\right)$ \\
\hline $0 \varepsilon \varepsilon " Z$ & & & & $0 \varepsilon \varepsilon Z$ & \\
\hline 0020 & & & $0 \cos ^{\prime} 0$ & & $\left(0 \mathrm{C} \varepsilon^{\circ} 0\right)$ \\
\hline 0000 & & & & & $000^{\circ} 0$ \\
\hline $611^{\prime} t$ & & & & & $6+16$ \\
\hline$\left(889^{\circ} 0\right)$ & $\varepsilon+L^{\prime} 0$ & & $\left(810^{\circ} 0\right)$ & & $(80,1)$ \\
\hline \multirow{2}{*}{\multicolumn{6}{|c|}{$000^{\circ} 0$}} \\
\hline & & & & & \\
\hline \multicolumn{6}{|l|}{$000^{\circ} 0$} \\
\hline \multicolumn{6}{|l|}{$000^{\prime} 0$} \\
\hline $990^{\circ} 0$ & & & & & 9900 \\
\hline \multirow{2}{*}{\multicolumn{6}{|c|}{$000^{\circ} 0$}} \\
\hline & & & & & \\
\hline $6 \angle 9^{\prime} 1$ & & $\left(296^{\circ} 0\right)$ & 97972 & & \\
\hline \multicolumn{6}{|l|}{$000^{\circ} 0$} \\
\hline \multicolumn{6}{|l|}{$000^{\prime} 0$} \\
\hline $800^{\circ} 0$ & $029^{\circ} 9$ & 2261 & $(0862)$ & & (609'9) \\
\hline \\
\hline \multicolumn{6}{|l|}{0000} \\
\hline \multirow{2}{*}{\multicolumn{6}{|c|}{$\frac{000^{\circ} 0}{000^{\circ} 0}$}} \\
\hline & & & & & \\
\hline $\begin{array}{l}17 \sigma_{1} \\
266 k \\
(w 9)\end{array}$ & $\begin{array}{l}\text { suOH:PPV } \\
\text { ocoss } \\
\text { (WS) }\end{array}$ & $\begin{array}{l}\text { uopleterasov } \\
\text { coss } \\
\text { (wis) }\end{array}$ & 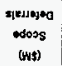 & 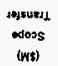 & $\begin{array}{l}\text { coppreac } \\
\text { odoss } \\
\text { (ont) }\end{array}$ \\
\hline & & VHO JАOOF & Iresषg LO & & \\
\hline
\end{tabular}

\begin{tabular}{|c|c|c|c|c|c|c|c|c|}
\hline $566^{\circ} 5$ & opts & BLS'L & $\left(920^{\circ} \bar{c}\right.$ & $\left(s s p^{\prime} 0\right)$ & 8LS:2 & & & $7 \forall 101$ \\
\hline 0000 & 0000 & 0000 & 0000 & $000^{\circ} 0$ & $000 \%$ & & $\angle E 0-56$ - INS & \\
\hline 0000 & 0000 & 0000 & $000^{\circ} 0$ & $(00 z 0)$ & $00 Z^{\prime} 0$ & & $000-96-3 \mathrm{NSS}$ & \\
\hline$\left(09^{\circ} 0\right)$ & $000^{\circ} 0$ & $000^{\circ} 0$ & $\left(\cos ^{\circ} 0\right)$ & $000^{\circ} 0^{\circ}$ & $000^{\circ} 0$ & & $560-96-3 N S$ & \\
\hline 0000 & $000 \% 0$ & 0000 & 0000 & 0000 & 000 & & $880-36 \cdot \mathrm{INS}$ & \\
\hline 0000 & 0000 & 0000 & $000^{\circ} 0$ & $000^{\circ} 0$ & 0000 & & $860-56-\mathrm{ANS}$ & \\
\hline$(0+1.0)$ & $089^{\circ} 0$ & $000^{\circ} 0$ & $(0<10)$ & $000^{\circ} 0$ & 0000 & & 1-10-S6-3NS & \\
\hline $000^{\circ} 0$ & 0000 & $000^{\prime} 0$ & 0000 & $000^{\circ} 0$ & $000^{\circ} 0$ & & $950-60 \mathrm{SNS}$ & \\
\hline $00 \varepsilon$ & LBLL & 0000 & 0000 & $(181.0)$ & $00 \varepsilon^{\circ} 0$ & & $120-96=\mathrm{NSS}$ & \\
\hline 0000 & 0000 & 0000 & $000^{\circ} 0$ & $000^{\circ} 0$ & 0000 & & $290-96-\mathrm{JNS}$ & \\
\hline 0000 & 0000 & 0000 & $000^{\circ} 0$ & $\left(9200^{\circ} 0\right)$ & $9 \angle 0^{\prime} 0$ & & $920-96=\mathrm{NS}$ & \\
\hline$(9 \mathrm{~s} 00)$ & 0000 & 0000 & 0000 & $000^{\prime} 0$ & $\left(990^{\circ} 0\right)$ & & $820-56-5 N \mid$ & \\
\hline 0000 & $000^{\circ} 0$ & 0000 & $000^{\prime} 0$ & $000^{\prime} 0$ & 0000 & & text-96-3N5 & \\
\hline $000^{\prime} 0$ & 0080 & $000^{\circ} 0$ & $000^{\prime} 0$ & $000^{\prime} 0$ & $\left(00 \varepsilon^{\circ} 0\right)$ & & $090^{0-96} \mathrm{-ANS}$ & in \\
\hline SLZ'E & 9981 & $\angle 96^{\circ} 0$ & $\left(9+9^{\prime} z\right)$ & $000^{\prime} 0$ & $620 \varepsilon$ & & $850-96-\mathrm{JSS}$ & In \\
\hline $000^{\circ} 0$ & 0000 & 0000 & 0000 & $000^{\prime} 0$ & $000^{\circ} 0$ & & $100-96-\mathrm{sNS}$ & +1 \\
\hline $000^{\circ} 0$ & 0000 & 0000 & 0000 & $000^{\circ} 0$ & $000^{\circ} 0$ & & 7 70096"-sNS & Fis \\
\hline 0000 & $929^{\circ} 0$ & $19 g^{\prime} 0$ & $\left(\mathrm{b60^{ \circ } 0}\right)$ & 0000 & $\left(880^{\circ} 1\right)$ & & Et0-969-3NS & in \\
\hline 0000 & $2 z g^{\circ} 0$ & $000^{\circ} 0$ & $000^{\circ} 0$ & $000^{\circ} 0$ & $\left(Z Z \mathrm{~s}^{\prime} 0\right)$ & & OLO-96-INS & int \\
\hline 0000 & $000^{\circ} 0$ & 0000 & $000^{\prime} 0$ & $000^{\prime} 0$ & 0000 & & 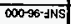 & i'th \\
\hline 2681 & 001.0 & 0000 & $\angle 18^{\circ} 0$ & 0000 & $926^{\circ} 0$ & & $500-96-\mathrm{JNS}$ & ini \\
\hline EOE'O & 92270 & 0000 & $\angle \angle L O$ & $000^{\prime} 0$ & $(0010)$ & 12ROKuEO S6A- & $200-96-3 \times 5$ & int \\
\hline $\begin{array}{l}1501 \\
966 t \\
(w 5)\end{array}$ & $\begin{array}{l}\text { suomppy } \\
\text { edoss } \\
\text { (Nt5) }\end{array}$ & 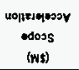 & $\begin{array}{l}\text { sienogen } \\
\text { cooss } \\
\text { (Ns) }\end{array}$ & $\begin{array}{l}\text { 10fsuti1 } \\
\text { odoss } \\
\text { (Ws) }\end{array}$ & $\begin{array}{l}\text { Lopmed } \\
\text { coss } \\
\text { (its) }\end{array}$ & 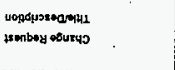 & on & 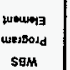 \\
\hline
\end{tabular}

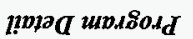

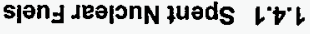

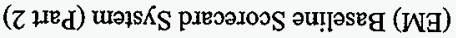

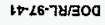




\begin{tabular}{|c|c|c|c|c|c|c|c|c|c|c|c|c|c|c|c|c|c|c|c|c|c|}
\hline (ISL't) & $\left(00 z^{2}\right)$ & $000^{\circ} 0$ & $000^{\prime} 0$ & $0,00^{\prime} 0$ & $000^{\circ} 0$ & {$\left[00 z^{2}\right)$} & GL9'Z & $000^{\circ} 0$ & $000^{\circ} 0$ & $g \angle \phi^{\prime} \boldsymbol{P}$ & $000 \%$ & $(0080)$ & $(9299)$ & $9 \angle 2 Z$ & 0000 & $(9 z 2 b)$ & 2100 & $\left(\varepsilon 61^{\circ}\right)$ & & & $7+101$ \\
\hline 0000 & 0000 & & & & & & 0000 & & & & & & 0000 & & & & & & & & \\
\hline $000^{\circ} 0$ & 000 & & & & & & $000^{\circ} 0$ & & & & & & 0000 & & & & & & & & \\
\hline 0000 & $000^{\circ} 0$ & & & & & & 0000 & & & & & & 0000 & & & & & & & & \\
\hline 0000 & 0000 & & & & & & 0000 & & & & & & 0000 & & & & & & & & \\
\hline $000^{\circ} 0$ & 0000 & & & & & & 0000 & & & & & & 0000 & & & & & & & & \\
\hline 0000 & $000^{\circ} 0$ & & & & & & 0050 & & & 0050 & & & $(\cos 0)$ & 0000 & 0000 & $\left(009^{\prime} 0\right)$ & $000^{\circ} 0$ & 0000 & & $200-a 6-0 \mathrm{MS}$ & \\
\hline $000^{\circ} 0$ & 0000 & & & & & & 0000 & & & & & & 0000 & 0900 & 0000 & 0000 & (Oتو0'0) & 0000 & & $810-96 \mathrm{ZSV}$ & \\
\hline$\left(00 \varepsilon^{\circ} 0\right)$ & $000^{\circ} 0$ & & & & & & 0000 & & & & & & $\left(0+\varepsilon^{\circ} 0\right)$ & 0000 & 0000 & $000^{\circ} 0$ & $000^{\circ} 0$ & $(0+8)$ & & S!l0-263-34 & \\
\hline 0001 & 0000 & & & & & & 0006 & & & $006 \%$ & & & 0000 & Doti & 0000 & $(00+1)$ & $000^{\circ} 0$ & $000^{\circ}$ & & $06,-969-5 \mathrm{~V}$ & \\
\hline 0000 & 0000 & & & & & & $000^{\circ} 0$ & & & & & & $000^{\circ} 0$ & 0080 & 0000 & $000^{\circ} 0$ & $\left(00 \varepsilon^{\circ} 0\right)$ & $000^{\prime} 0$ & & $600-969 \mathrm{sy}$ & \\
\hline 0000 & 0000 & & & & & & $000^{\circ} 0$ & & & & & & 0000 & $981^{\circ} 0$ & $000^{\circ} 0$ & $000^{\prime} 0$ & $(9810)$ & 0000 & & $800-863-\mathrm{SV}$ & \\
\hline $000^{\circ} 0$ & 0000 & & & & & & $000^{\circ} 0$ & & & & & & $000^{\circ} 0$ & 0080 & 0000 & 0000 & $8 \operatorname{ss}^{\circ} 0$ & $\left(\varepsilon \mathcal{G S}^{\circ} 0\right)$ & & $200-969-54$ & \\
\hline (SELO) & $000^{\circ} 0$ & & & & & & $g L S^{\prime} Z$ & & & GLGZ & & & (008) & $00 z^{\prime} 0$ & & $\left(00 S^{2} 2\right)$ & & $(000 \%$ & & $900-560.54$ & Istit \\
\hline+190 & 0000 & & & & & & 0000 & & & & & & t.:G 0 & $0+\varepsilon^{\prime} 0$ & 0000 & 7210 & $000^{\circ} 0$ & $000^{\prime} 0$ & 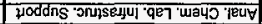 & $200-369-54$ & $|\leq|$ \\
\hline$(0009)$ & $(00 \mathrm{CL})$ & & & & & (0021) & $\left(0,08^{\prime 2}\right)$ & & & & & $(\overline{O O B} 1)$ & $(0002)$ & & & & & $\left(000^{\circ} z\right)$ & 7. & roo-963-Sv & givisi \\
\hline $\begin{array}{c}\text { Tw101 } \\
\text { op } \\
\text { (ws) }\end{array}$ & $\begin{array}{l}\text { inol } \\
\text { (160) } \\
(n \neq 1)\end{array}$ & $\begin{array}{c}\text { suopppy } \\
\text { woss } \\
\text { (Ws) }\end{array}$ & $\begin{array}{c}\text { 4operemosy } \\
\text { coss } \\
\text { (int) }\end{array}$ & 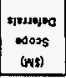 & $\begin{array}{c}\text { Imtures } \\
\text { edoos } \\
\text { (ins) }\end{array}$ & 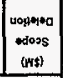 & $\begin{array}{l}1001 \\
1061 \\
\text { (w) } \$\end{array}$ & $\begin{array}{l}\text { shoptppy } \\
\text { octoss } \\
\text { (Wt) }\end{array}$ & 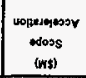 & 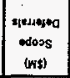 & 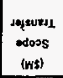 & $\begin{array}{l}\text { uoppenod } \\
\text { odors } \\
\text { (WF) }\end{array}$ & $\begin{array}{l}\text { imol } \\
9656 \\
(k s)\end{array}$ & $\begin{array}{l}\text { suopuppr } \\
\text { ofoss } \\
\text { (ws) }\end{array}$ & $\begin{array}{l}\text { uogneterossy } \\
\text { Nojs } \\
\text { (ws) }\end{array}$ & 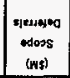 & $\begin{array}{l}\text { Jogstus } \\
\text { odoss } \\
\text { (N) }\end{array}$ & 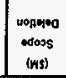 & 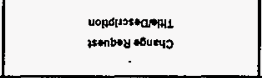 & OON & 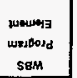 \\
\hline
\end{tabular}

\section{1p1a $u p i s 0 \lambda_{d}$}




\begin{tabular}{|c|c|c|c|c|c|c|}
\hline$Z 16^{\prime} 0$ & $000^{\circ} 0$ & $000^{\circ} 0$ & $000^{\circ} 0$ & $000^{\prime} 0$ & $000^{\circ} 0$ & $0000^{\circ}$ \\
\hline $000^{\circ}$ & 0000 & & & & & \\
\hline $000^{\circ} 0$ & 0000 & & & & & \\
\hline 0000 & $000^{\circ} 0$ & & & & & \\
\hline OSt 0 & 0000 & & & & & \\
\hline $29 L^{\circ} 0$ & $000^{\prime} 0$ & & & & & \\
\hline $\begin{array}{c}7 \text { wol } \\
\text { \&D } \\
\text { (wt) }\end{array}$ & $\begin{array}{l}1001 \\
9661 \\
\text { (W:5) }\end{array}$ & $\begin{array}{l}\text { Suopppp } \\
\text { odors } \\
\text { (WHS) }\end{array}$ & 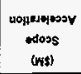 & $\begin{array}{l}\text { Menweoc } \\
\text { cooss } \\
\text { (ws) }\end{array}$ & $\begin{array}{l}\text { doysutest } \\
\text { edors } \\
\text { (ws) }\end{array}$ & 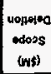 \\
\hline
\end{tabular}

\begin{tabular}{|c|c|c|c|c|c|}
\hline 0000 & $000^{\circ} 0$ & $000^{\circ} 0$ & $000^{\circ} 0$ & $000^{\circ} 0$ & $000^{\circ} 0$ \\
\hline 0000 & & & & & \\
\hline \multicolumn{6}{|l|}{$000^{\circ} 0$} \\
\hline \multicolumn{6}{|l|}{$000^{\circ} 0$} \\
\hline \multirow{2}{*}{\multicolumn{6}{|c|}{$000^{\circ} 0$}} \\
\hline & & & & & \\
\hline $\begin{array}{l}1401 \\
256 ! \\
(K \$)\end{array}$ & $\begin{array}{l}\text { suopuppy } \\
\text { edoss } \\
\text { (ws) }\end{array}$ & $\begin{array}{l}\text { coppesposory } \\
\text { cdoss } \\
\text { (WHs) }\end{array}$ & 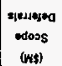 & $\begin{array}{l}\text { sogsules } 1 \\
\text { Nors } \\
\text { (W:) }\end{array}$ & 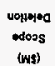 \\
\hline
\end{tabular}

\begin{tabular}{|c|c|c|c|c|c|c|c|c|}
\hline $216^{\circ} 0$ & OSLO & 0000 & 2920 & 0,000 & $000^{\circ} 0$ & & & $7 \forall 101$ \\
\hline 0000 & & & & & & & & \\
\hline 0000 & & & & & & & & \\
\hline 0000 & & & & & & & & \\
\hline OSL"0 & 0910 & & & & & 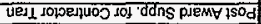 & म10-96.18 & ZS' \\
\hline 2920 & & & $29 \angle 0$ & & & 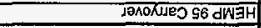 & $200-96-10 y$ & |हs' \\
\hline $\begin{array}{l}1701 \\
9661 \\
\text { (H+s) }\end{array}$ & $\begin{array}{l}\text { suopppy } \\
\text { wexs } \\
\text { (4w:s) }\end{array}$ & 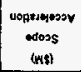 & $\begin{array}{l}\text { s]enesed } \\
\text { ocoss } \\
\text { (Wts) }\end{array}$ & $\begin{array}{l}\text { sigsuens } \\
\text { ocoss } \\
\text { (ws) }\end{array}$ & 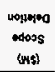 & 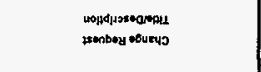 & סדי & 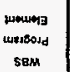 \\
\hline
\end{tabular}

\section{lipiog wpisoid}

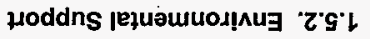

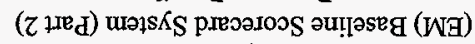

16-26-78/500 
DOE/RL.97-41

(EM) Baseline Scorecard System (Part 2)

Program Detail

\subsubsection{RCRA \& Operational Monitoring}

\begin{tabular}{|c|c|c|c|c|c|c|c|c|}
\hline & & & & FY1 & OS BASEUI & SCOPE CHAN & & $\therefore$ \\
\hline $\begin{array}{l}\text { Wes } \\
\text { Program } \\
\text { Elorment }\end{array}$ & CR No. & $\begin{array}{l}\text { Change Roquest } \\
\text { Ttterioesctiption }\end{array}$ & $\begin{array}{c}\text { (SW) } \\
\text { Sespe } \\
\text { Dontion }\end{array}$ & \begin{tabular}{|l|} 
(sth) \\
seopt \\
Transtor
\end{tabular} & $\begin{array}{c}\text { (SM) } \\
\text { Scope } \\
\text { Dofortsils }\end{array}$ & $\begin{array}{c}\text { (stin) } \\
\text { Scope } \\
\text { Accaloration }\end{array}$ & $\begin{array}{c}\text { (sMin) } \\
\text { Scope } \\
\text { Additions }\end{array}$ & $\begin{array}{l}\text { (5M) } \\
\text { 1996 } \\
\text { Tatal }\end{array}$ \\
\hline 1.5 .3 & RLEO6-001 & FY 1996 Re-Baseline & $(3.438)$ & & 2.915 & & & $(0.523)$ \\
\hline 1.5 .3 & $R<$ E96-004 & Midyear Rebaseline & & 0.200 & & & & 0.200 \\
\hline & & & & & & & & 0.000 \\
\hline$\because$ & & & & & & & & 0.000 \\
\hline & & & & & & & & 0.000 \\
\hline TOTAL & & & (3.438) & 0.200 & 2.915 & 0.000 & 0.000 & $(0.323)$ \\
\hline
\end{tabular}

\begin{tabular}{|c|c|c|c|c|c|}
\hline \multicolumn{6}{|c|}{ FY 1997 SASELINE SCOPE CHANGES } \\
\hline $\begin{array}{c}\text { (SH) } \\
\text { Scope } \\
\text { Dethitlon }\end{array}$ & $\begin{array}{c}\text { (SMM) } \\
\text { Scopt } \\
\text { Trenster }\end{array}$ & $\begin{array}{c}\text { (5MI) } \\
\text { Scopo } \\
\text { Defereralis }\end{array}$ & $\begin{array}{c}(\mathrm{sm}) \\
\text { Scope } \\
\text { Acceleration }\end{array}$ & $\begin{array}{c}\text { (sim) } \\
\text { Scope } \\
\text { Additions }\end{array}$ & $\begin{array}{l}\text { (5M) } \\
1997 \\
\text { Total }\end{array}$ \\
\hline & & 2.309 & & & 2.309 \\
\hline & & & & & 0.000 \\
\hline & & & & & 0.000 \\
\hline & & & & & 0.000 \\
\hline & & & & & 0.000 \\
\hline 0.000 & 0.000 & 2.309 & 0.00 & 0.000 & 2.309 \\
\hline
\end{tabular}

\begin{tabular}{|c|c|c|c|c|c|c|}
\hline \multicolumn{6}{|c|}{ FY 19DE BASELANE SCOPE CHANGES } & \multirow[b]{2}{*}{$\begin{array}{l}\text { (SMM) } \\
\text { CR } \\
\text { TOIAL }\end{array}$} \\
\hline $\begin{array}{c}(5 M) \\
\text { Scose } \\
\text { Detintion }\end{array}$ & $\begin{array}{l}\text { (sm)- } \\
\text { Seope } \\
\text { Transtor }\end{array}$ & $\begin{array}{c}\text { (sMm) } \\
\text { Seope } \\
\text { Doformals }\end{array}$ & $\begin{array}{c}\text { (SM) } \\
\text { Scope } \\
\text { Accolentition }\end{array}$ & $\begin{array}{c}\text { (5M) } \\
\text { Seope } \\
\text { Additions }\end{array}$ & $\begin{array}{l}\text { (15) } \\
\text { 1996 } \\
\text { Total }\end{array}$ & \\
\hline & & & & & 0.000 & 1.786 \\
\hline & & & & & 0.000 & 0.200 \\
\hline & & & & & 0.000 & 0.000 \\
\hline & & & & & 0.000 & 0.000 \\
\hline & & & & & 0.000 & 0.000 \\
\hline 0.000 & 0.000 & 0.000 & 0.000 & 0.000 & 0.000 & 1.988 \\
\hline
\end{tabular}



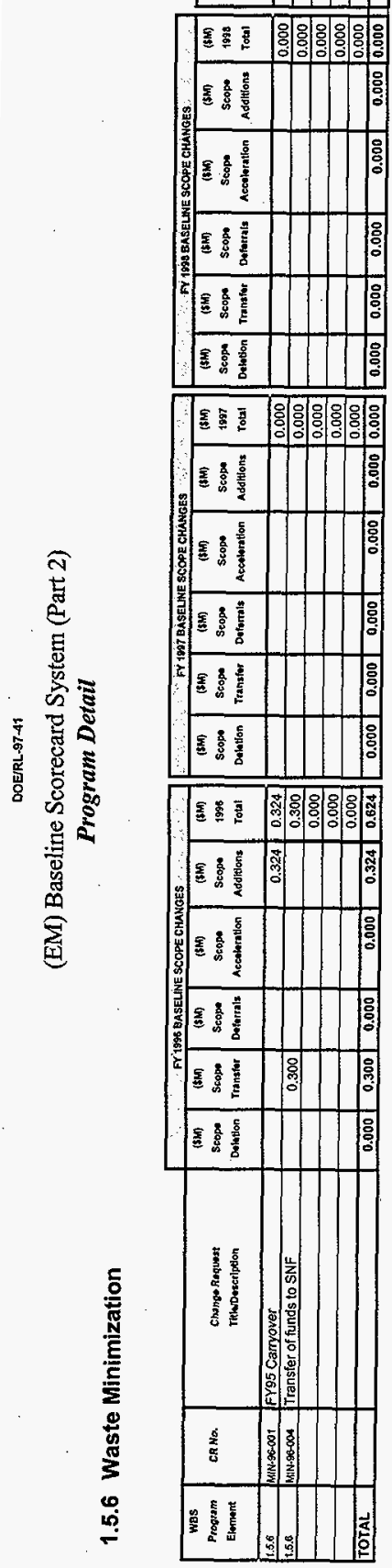


\begin{tabular}{|c|c|c|c|c|c|c|}
\hline$\ldots$ & $\ldots$ & $000^{\circ} 0$ & $000^{\circ} 0$ & $000^{\circ} 0$ & ooth & $000^{\circ} 0$ \\
\hline $000^{\circ} 0$ & 0000 & & & & & \\
\hline $000^{\circ} 0$ & $000^{\circ} 0$ & & & & & \\
\hline$\left(\mathcal{G} b 0^{\circ} 0\right)$ & $000^{\circ} 0$ & & & & & \\
\hline$\left(96 \varepsilon^{\circ} 0\right)$ & $000^{\circ} 0$ & & & & & \\
\hline $08 Z^{\circ} 9$ & $00 \mathrm{p}^{\circ} \varepsilon$ & & & & $00 t \varepsilon$ & \\
\hline (8080) & $000^{\circ}$ & & & & & \\
\hline $080^{\circ} 0$ & $000^{\circ}$ & & & & & \\
\hline 1000 & $000^{\circ} 0$ & & & & & \\
\hline$\angle 20^{\circ} 0$ & 000 & & & & & \\
\hline $890^{\circ} 0$ & $000^{\circ} 0$ & & & & & \\
\hline $980^{\circ} 0$ & $000^{\circ} 0$ & & & & & \\
\hline $810^{\circ} 0$ & $000^{\circ}$ & & & & & \\
\hline$\left(980^{\circ} 0\right)$ & 0000 & & & & & \\
\hline 开\#H & 0008 & & & & 0008 & \\
\hline $901 \%$ & 0000 & & & & & \\
\hline $298^{\circ} 0$ & 0000 & & & & & \\
\hline $\begin{array}{l}\text { Trod } \\
\text { yo } \\
\text { twst }\end{array}$ & $\begin{array}{l}1701 \\
1685 \\
(143)\end{array}$ & $\begin{array}{l}\text { suoplppy } \\
\text { odoss } \\
\text { (ws) }\end{array}$ & $\begin{array}{l}\text { 4operoposy } \\
\text { udoos } \\
\text { (W:s) }\end{array}$ & 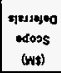 & $\begin{array}{l}\text { rogsuras } \\
\text { iclooss } \\
\text { (wis) }\end{array}$ & $\begin{array}{l}\text { nophen } \\
\text { adoss } \\
\text { (WE) }\end{array}$ \\
\hline
\end{tabular}

\begin{tabular}{|c|c|c|c|c|c|}
\hline$\$ 202$ & $000^{\circ} 0$ & $\left(810^{\circ} 0\right)$ & $\angle 80^{\circ} 0$ & $000^{\circ} \mathrm{L}$ & $000^{\circ} 0$ \\
\hline \multicolumn{6}{|l|}{0000} \\
\hline \multicolumn{6}{|l|}{$000^{\prime} 0$} \\
\hline \multicolumn{6}{|l|}{0000} \\
\hline \multicolumn{6}{|l|}{$000^{\circ} 0$} \\
\hline $008^{\prime} \mathrm{Z}$ & & & & $008 \mathrm{Z}$ & \\
\hline \multicolumn{6}{|l|}{0000} \\
\hline \multicolumn{6}{|l|}{0000} \\
\hline $620^{\circ} 0$ & & $(\varepsilon 100)$ & $\angle 80^{\circ} 0$ & & \\
\hline \multicolumn{6}{|l|}{0000} \\
\hline \multicolumn{6}{|l|}{0000} \\
\hline \multicolumn{6}{|l|}{$000^{\prime} 0$} \\
\hline \multirow{2}{*}{\multicolumn{6}{|c|}{$000^{\circ} 0$}} \\
\hline & & & & & \\
\hline \multicolumn{4}{|l|}{$00 z^{2} b$} & 0020 & \\
\hline \multicolumn{6}{|l|}{$000^{\circ} 0$} \\
\hline \multicolumn{6}{|l|}{$000^{\circ} 0$} \\
\hline $\begin{array}{l}\text { InOL } \\
2661 \\
\text { (W:5) }\end{array}$ & $\begin{array}{l}\text { tuogpppy } \\
\text { edors } \\
\text { (wt) }\end{array}$ & 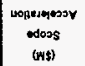 & $\begin{array}{l}\text { stereysen } \\
\text { edors } \\
\text { (ws) }\end{array}$ & $\begin{array}{l}\text { deysures } \\
\text { Wdors } \\
\text { (Ws) }\end{array}$ & $\begin{array}{l}\text { uopiens } \\
\text { edors } \\
\text { (Ws) }\end{array}$ \\
\hline & & 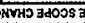 & 77asYa 16 & of AS : & \\
\hline
\end{tabular}

\begin{tabular}{|c|c|c|c|c|c|c|c|c|}
\hline $189^{\circ} 0$ & $0899^{\prime} \varepsilon$ & 2100 & ISL'L & $\left(s+L^{\prime} 0\right.$ & $\left(29 p^{\prime} \varepsilon\right)$ & & & $7 \forall 101$ \\
\hline \multicolumn{9}{|l|}{$000^{\circ} 0$} \\
\hline & & & & & & & & \\
\hline$(s+0)$ & (stoo) & & & & & & 25096 - Fimd & \\
\hline$(9680)$ & & & & & $(9680)$ & & $520-66 \% \mathrm{WMd}$ & \\
\hline 0800 & & & $080^{\circ} 0$ & & & & $800-96$-FMd & \\
\hline$\left(80 \varepsilon_{0}\right)$ & & & & $\left(80 \varepsilon^{\circ} 0\right)$ & & & $060-96+1$ WMS & \\
\hline 0800 & 0800 & & & & & & $620-96-5 \mathrm{WMd}$ & \\
\hline$\left(\varepsilon 20^{\circ} 0\right)$ & & 2100 & $\left(s 0^{\circ} 0\right)$ & & & & B20-96-FMA & \\
\hline$\angle 200$ & & & & $\angle \angle 0^{\circ} 0$ & & & $320-56+14 M$ & \\
\hline $890^{\circ} 0$ & 8500 & & & & & & $520-96.5 / M d$ & \\
\hline $980^{\circ} 0$ & $980^{\circ} 0$ & & & & & & $020-96$ - WiMd & \\
\hline 8100 & 8100 & & & & & & 21006 Fima & \\
\hline$\left(980^{\circ} 0\right)$ & $0 Z Z^{\prime} 0$ & & & $80 \overline{80}$ & $(t+90)$ & & ol0-56-YWhd & \\
\hline$(2 Z 80)$ & & & & $\left(Z z 8^{\circ} 0\right)$ & & & $80096 \% \mathrm{KMd}$ & \\
\hline $901 \%$ & & & $90 L^{-1}$ & & & & 이 166 서 & 52 \\
\hline $298^{\circ} 0$ & $6 L \varepsilon \varepsilon$ & & & & $(29+2)$ & 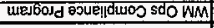 & roos: $\mathrm{kHMd}$ & 526 \\
\hline $\begin{array}{l}10101 \\
9661 \\
(N 5)\end{array}$ & $\begin{array}{l}\text { suopppy } \\
\text { cooss } \\
\text { (ws) }\end{array}$ & $\begin{array}{l}\text { Uopteruesory } \\
\text { edoss } \\
\text { (wis) }\end{array}$ & 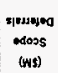 & $\begin{array}{l}\text { Hofsuts! } \\
\text { edoos } \\
\text { (ws) }\end{array}$ & 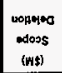 & 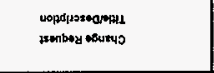 & ס4 & $\begin{array}{l}\text { purcuef } \\
\text { turisosd } \\
\text { sam }\end{array}$ \\
\hline
\end{tabular}

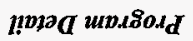

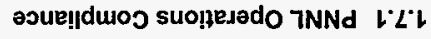

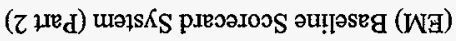


(EM) Baseline Scorecard System (Part 2)

Program Detail

\subsubsection{PNNL Hanford Mission}

\begin{tabular}{|c|c|c|c|c|c|c|c|c|}
\hline \multirow[b]{2}{*}{$\begin{array}{l}\text { Was } \\
\text { Progtam } \\
\text { Elorment }\end{array}$} & \multirow[b]{2}{*}{ CR No. } & \multirow[b]{2}{*}{$\begin{array}{l}\text { Change Request } \\
\text { Trisedoseription }\end{array}$} & \multicolumn{6}{|c|}{ FY 1996 BASELNE SCOPE CHANGES } \\
\hline & & & $\begin{array}{l}\text { (5:M) } \\
\text { Seope } \\
\text { Dowetion }\end{array}$ & \begin{tabular}{c|} 
(smins \\
seopes \\
Transfor
\end{tabular} & 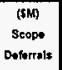 & $\begin{array}{c}\text { (5MM) } \\
\text { Scops } \\
\text { Accositraton }\end{array}$ & $\begin{array}{c}\text { (\$M) } \\
\text { Scops } \\
\text { Additilons }\end{array}$ & $\begin{array}{l}\text { (5N) } \\
1996 \\
\text { Tetol }\end{array}$ \\
\hline 1.72 .1 .1 .22 & FWM-96-012 & Biodiversity Study & & & & & 0.000 & 0.000 \\
\hline 1.7 .2 & PWM-98-006 & Pub. Safety \& Resource Protect Prog & $(0.221)$ & $(0.020)$ & 0.175 & 0.075 & 0.333 & 0.342 \\
\hline 1.7 .2 & PWM-95-ac3 & & & & & & 0.248 & 0.248 \\
\hline 1.7 .2 & PWM-95-034 & & & $(0.050)$ & & & & $(0.050)$ \\
\hline & PWM-95-039 & & & & & & & 0.000 \\
\hline & & & & & & & & 0.000 \\
\hline & & & & & & & & 0.000 \\
\hline TOTAL & & & $(0.221)$ & $(0.070)$ & 0.175 & 0.075 & 0.581 & 0.540 \\
\hline
\end{tabular}

\begin{tabular}{|c|c|c|c|c|c|}
\hline \multicolumn{6}{|c|}{ FY 1997 BASELLAFE SCOPEE CHANGES } \\
\hline $\begin{array}{c}\text { (SM) } \\
\text { Scops } \\
\text { Dention }\end{array}$ & $\begin{array}{c}\text { (SN) } \\
\text { Seope } \\
\text { Transtor }\end{array}$ & $\begin{array}{c}\text { (SiM) } \\
\text { Seope } \\
\text { Dotomale }\end{array}$ & $\begin{array}{c}\text { (\$H) } \\
\text { Scopo } \\
\text { Acecenration }\end{array}$ & $\begin{array}{c}\text { (SM) } \\
\text { Scope } \\
\text { Additions }\end{array}$ & $\begin{array}{l}\text { (5im) } \\
\text { Tos? } \\
\text { Total }\end{array}$ \\
\hline & & & & & 0.000 \\
\hline & & & & & 0.000 \\
\hline & & & & & 0.000 \\
\hline & & & & & 0.000 \\
\hline & & 0.050 & & 0.580 & 0.630 \\
\hline & & & & & 0.000 \\
\hline & & & & & 0.000 \\
\hline 0.000 & 0.000 & 0.050 & 0.000 & 0.580 & 0.630 \\
\hline
\end{tabular}

\begin{tabular}{|c|c|c|c|c|c|c|}
\hline & & DBEASEL & E SCOPE CHA & & & \\
\hline $\begin{array}{c}\text { (\$M) } \\
\text { Scope } \\
\text { Delotion }\end{array}$ & $\begin{array}{c}\text { (SW) } \\
\text { Seope } \\
\text { Transwh }\end{array}$ & $\begin{array}{c}(A M) \\
\text { Seope } \\
\text { Detarrats }\end{array}$ & $\begin{array}{c}\text { (S1M) } \\
\text { Seope } \\
\text { Accolorntion }\end{array}$ & $\begin{array}{c}\text { (\$in) } \\
\text { Scopo } \\
\text { Additions }\end{array}$ & $\begin{array}{l}\text { (5s) } \\
1988 \\
\text { rosis }\end{array}$ & $\begin{array}{c}(\mathrm{sM}) \\
\mathrm{CR} \\
\text { TOTAL, }\end{array}$ \\
\hline & & & & & 0.000 & 0.000 \\
\hline & & & & & 0.000 & 0.342 \\
\hline & & & & & 0.000 & 0.248 \\
\hline & & & & & 0.000 & 0.050 \\
\hline & & & & & 0.000 & 0.630 \\
\hline & & & & & 0.000 & 0.000 \\
\hline & & & & & 0.000 & 0.000 \\
\hline 0,000 & 0.000 & 0.000 & 0.000 & 0.000 & 0,000 & 1.170 \\
\hline
\end{tabular}




\begin{tabular}{|c|c|c|c|c|c|c|}
\hline $0 \varepsilon^{\prime} z z$ & $00^{\prime} 0$ & 000 & $00^{\circ} 0$ & $00^{\circ} 0$ & $00^{\circ} 0$ & $00^{\prime} 0$ \\
\hline $00^{\prime} 0$ & $00^{\circ} 0$ & & & & & \\
\hline $00^{\prime} 0$ & 000 & & & & $\therefore$ & \\
\hline $00^{\prime} 0$ & $00^{\circ} 0$ & & & & & \\
\hline $00^{\circ} 0$ & $00^{\circ} 0$ & & & & & \\
\hline $0 \varepsilon^{\prime} z z$ & $00^{\prime} 0$ & & & & & \\
\hline $\begin{array}{l}\text { T10s } \\
\text { \&o } \\
\text { (WS) }\end{array}$ & $\begin{array}{l}\text { In:1 } \\
\text { 85st } \\
\text { (wSI) }\end{array}$ & $\begin{array}{l}\text { suop:pPY } \\
\text { edoos } \\
\text { (WHS) }\end{array}$ & 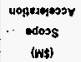 & 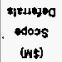 & $\begin{array}{l}\text { ongsues } \\
\text { odoss } \\
\text { (kst) }\end{array}$ & $\begin{array}{l}\text { Uopored } \\
\text { odoss } \\
\text { (WH) }\end{array}$ \\
\hline
\end{tabular}

\begin{tabular}{|c|c|c|c|c|c|}
\hline $00^{\circ} 0$ & $100^{\circ} 0$ & $00^{\prime} 0$ & $00^{\circ} 0$ & $00^{\prime} 0$ & $00^{\circ} 0$ \\
\hline \multicolumn{6}{|l|}{$00^{\circ} 0$} \\
\hline \multicolumn{6}{|l|}{$00^{\circ} 0$} \\
\hline \multicolumn{6}{|l|}{$00^{\circ} 0$} \\
\hline \multirow{2}{*}{\multicolumn{6}{|c|}{$\frac{00^{\circ} 0}{00^{\circ} 0}$}} \\
\hline & & & & & \\
\hline $\begin{array}{l}\text { 1*1 } \\
266 ! \\
\text { (vist) }\end{array}$ & $\begin{array}{l}\text { suoptppy } \\
\text { ocoss } \\
\text { (WSs) }\end{array}$ & $\begin{array}{l}\text { uoninwososy } \\
\text { woss } \\
\text { (ws) }\end{array}$ & 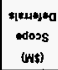 & $\begin{array}{l}\text { Hogsures } \\
\text { caoss } \\
\text { [WsI }\end{array}$ & 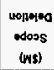 \\
\hline & & WHO ב ב.00 & ressvg & & \\
\hline
\end{tabular}

\begin{tabular}{|c|c|c|c|c|c|c|c|c|}
\hline $0 \varepsilon z 2$ & $0 \varepsilon z z$ & 00.0 & 000 & 000 & $00^{\circ} 0$ & & & $7 \forall 101$ \\
\hline 000 & & & & & & & & \\
\hline $00^{\circ} 0$ & & & & & & & & \\
\hline 000 & & & & & & & & \\
\hline $00^{\circ} 0$ & & & & & & & & \\
\hline $0 \varepsilon z 2$ & $0 \varepsilon 22$ & & & & & uonsueJ1 OWHd & & \\
\hline $\begin{array}{l}1901 \\
966 s \\
\text { (NS) }\end{array}$ & $\begin{array}{l}\text { SUOHHPD } \\
\text { COSS } \\
\text { (N\$) }\end{array}$ & 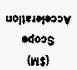 & $\begin{array}{l}\text { F|eriped } \\
\text { odoss } \\
\text { (Ws) }\end{array}$ & $\begin{array}{l}\text { seyturt1 } \\
\text { Noloss } \\
\text { (W\%) }\end{array}$ & $\begin{array}{l}\text { 4opkwa } \\
\text { edess } \\
\text { (wts) }\end{array}$ & 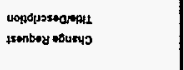 & बN म० & 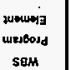 \\
\hline
\end{tabular}

\section{1ma}

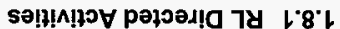

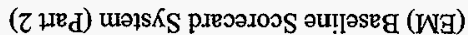




\begin{tabular}{|c|c|c|c|c|c|c|}
\hline (zotz) & 0000 & $000^{\circ} 0$ & $000^{\circ} 0$ & 0000 & $000^{\prime} 0$ & 0000 \\
\hline $002 \%$ & $000^{\circ} 0$ & & & & & \\
\hline OZLO 0 & 0000 & & & & & \\
\hline DOE० & $000^{\circ} 0$ & & & & & \\
\hline $000 \%$ & 0000 & & & & & \\
\hline $080^{\prime} 0$ & 000 & & & & & \\
\hline $000^{\prime} 0$ & $000 \%$ & & & & & \\
\hline$\left(206^{\prime} z\right)$ & $000^{\prime 0}$ & & & & & \\
\hline $\begin{array}{c}\text { Tw101 } \\
\text { yo } \\
\text { (w:) }\end{array}$ & $\begin{array}{l}\text { Imol } \\
8561 \\
\text { (W5) }\end{array}$ & $\begin{array}{l}\text { suopppy } \\
\text { odoss } \\
\text { (Wt) }\end{array}$ & 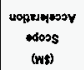 & 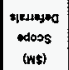 & $\begin{array}{l}\text { monsuest } \\
\text { odoss } \\
\text { (Kย) }\end{array}$ & $\begin{array}{l}\text { Uoperes } \\
\text { ocoss } \\
\text { (ㅂ) }\end{array}$ \\
\hline & \multicolumn{6}{|c|}{ SGONVH 3 3 } \\
\hline
\end{tabular}

\begin{tabular}{|c|c|c|c|c|c|}
\hline $000^{\circ} 0$ & 0000 & $000^{\circ} 0$ & $000^{\circ} 0$ & $000^{\circ} 0$ & $000^{\circ} 0$ \\
\hline \multicolumn{6}{|l|}{$000^{\prime} 0$} \\
\hline \multicolumn{6}{|l|}{0000} \\
\hline \multicolumn{6}{|l|}{$000^{\circ}$} \\
\hline \multicolumn{6}{|l|}{$000^{\circ} 0$} \\
\hline \multicolumn{6}{|l|}{$000^{\circ} 0$} \\
\hline \multirow{2}{*}{\multicolumn{6}{|c|}{$\begin{array}{l}000^{\prime} 0 \\
000^{\circ}\end{array}$}} \\
\hline & & & & & \\
\hline $\begin{array}{l}1701 \\
2665 \\
(N) 5)\end{array}$ & $\begin{array}{l}\text { suo|fppy } \\
\text { odoss } \\
\text { (WS) }\end{array}$ & $\begin{array}{c}\text { чoproqossy } \\
\text { moss } \\
\text { (wis) }\end{array}$ & 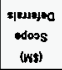 & 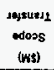 & $\begin{array}{l}\text { uonpwos } \\
\text { ecoss } \\
\text { (WSs) }\end{array}$ \\
\hline
\end{tabular}

\begin{tabular}{|c|c|c|c|c|c|c|c|c|}
\hline (z0z'z) & 0020 & 0000 & $000^{\circ} 0$ & $000^{\circ} 0$ & $(\mathrm{ZOB} Z \mathrm{Z})$ & & & $7 \forall 101$ \\
\hline 0020 & 0020 & & & & & & $820-96-21$ & \\
\hline OZL 0 & $0 Z 1 \cdot 0$ & & & & & - & $220-96-2 k$ & \\
\hline $00 \% 0$ & $00 \varepsilon^{\prime \prime 0}$ & & & & & & i20-86-2A & \\
\hline $000^{\circ} 0$ & $000^{\circ} 0$ & & & & & 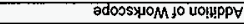 & $800-96-2 \lambda$ & $9+286$ \\
\hline 0800 & $080^{\circ} 0$ & & & & & Euppoday yWقg & $200-96-2 \lambda$ & 28'th \\
\hline $000 \%$ & $000 \%$ & & & & & ald-7망 of pod & $800-96-2 \lambda$ & I'ZE' \\
\hline$(206 z)$ & & & & & $(206 Z)$ & 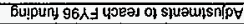 & 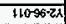 & 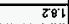 \\
\hline $\begin{array}{l}1001 \\
965 k \\
(w 9)\end{array}$ & $\begin{array}{l}\text { suoppppy } \\
\text { odess } \\
\text { (1ws) }\end{array}$ & $\begin{array}{c}\text { vopenyesy } \\
\text { woos } \\
\text { (wit) }\end{array}$ & 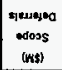 & $\begin{array}{l}\text { Instust } \\
\text { ecoss } \\
\text { (ins) }\end{array}$ & $\begin{array}{l}\text { vogesno } \\
\text { cososs } \\
(W \mid s)\end{array}$ & 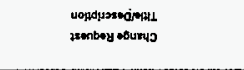 & סN Y כ & 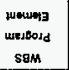 \\
\hline
\end{tabular}

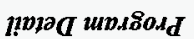

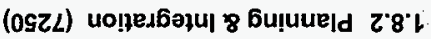

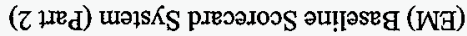

เพ- $26-74=00$ 

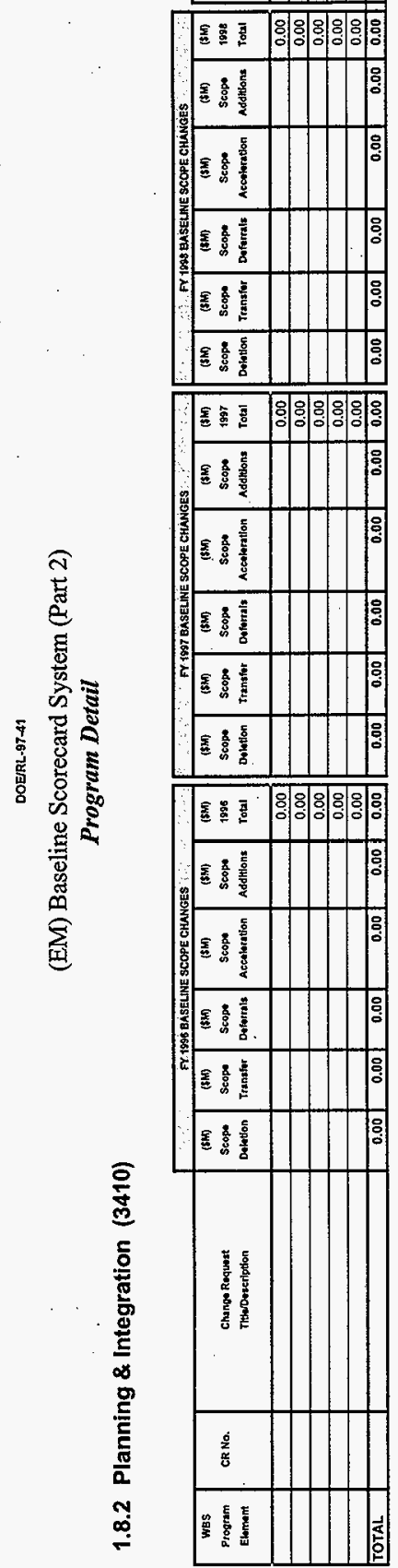

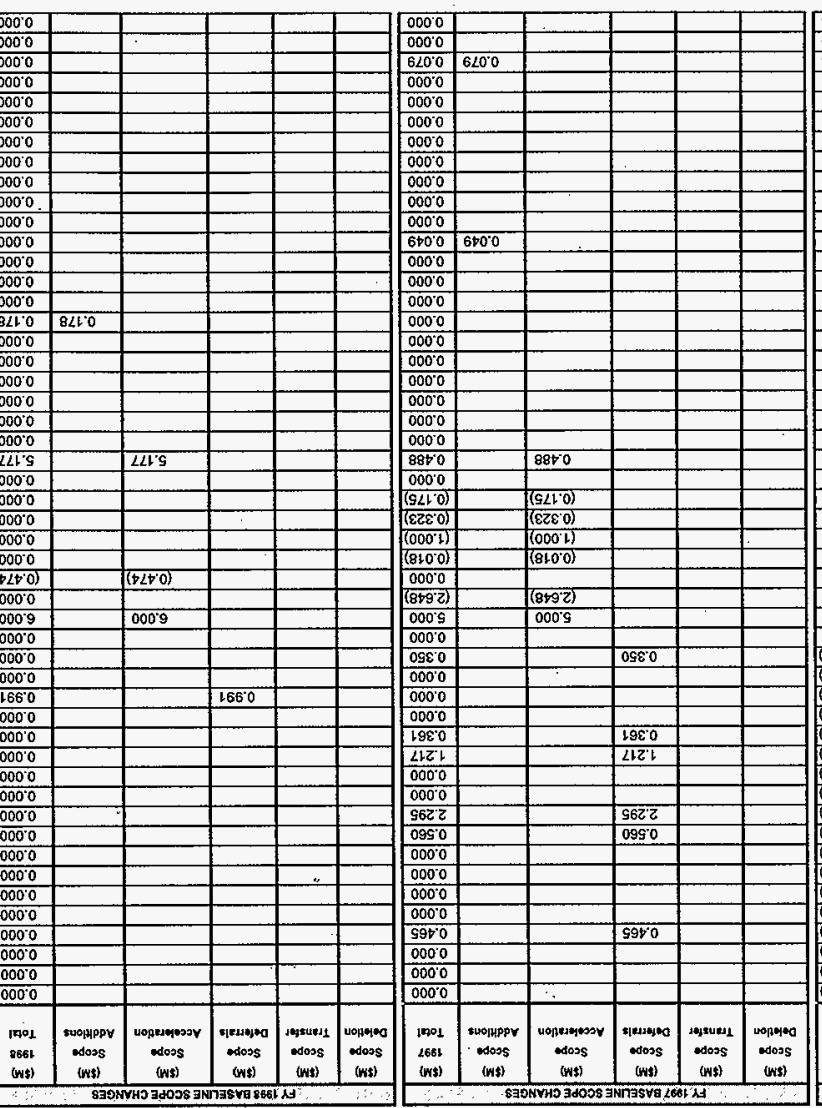

\begin{tabular}{|c|c|c|c|c|c|c|c|c|}
\hline हाZZ० & ELZZO & & & & & SUMOPYHEM rapU!MUMOG & 66090.d06 & 02 \\
\hline $08 \angle 2 \circ 0$ & $0640^{\circ} 0$ & & & & & 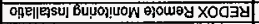 & $06096-408$ & 02 \\
\hline $8 \varepsilon+0^{\circ} 0$ & $8 \varepsilon+0^{\circ} 0$ & & & & & 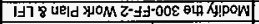 & 96096-408 & 02 \\
\hline $08+0^{\circ} 0$ & $08+0^{\prime} 0$ & & & & & 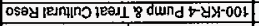 & c8090.40 & $0=2$ \\
\hline $0+200^{\circ} 0$ & & & & & $0 \neq 200$ & on!ppe sof juewaj!nbay SAJ Z-dñ-00Z & 18096-d0五 & 0 记 \\
\hline 08200 & 08200 & & & & & 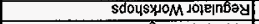 & 08036-d28 & 07 \\
\hline$\square . \angle 20$ & ILLE'0 & & & & & 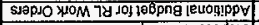 & $92096-\mathrm{d} 08$ & 02 \\
\hline 96160 & 96160 & & & & & $y-z|z|$ le dojsduna jo jesods!a & $5<056 \cdot d 38$ & 02 \\
\hline $29+10$ & Ż̈blo & & & & & sulseg LongodeAG JejOS H-E8: & $\varepsilon<005 \cdot d \supset$ & 02 \\
\hline $09 \angle 0^{\circ} O$ & $09 \angle 00$ & & & & & 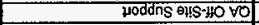 & $89095-d \partial 2$ & $0 \mathrm{z}$ \\
\hline $06+0 \% 0$ & $06+000$ & & & & & 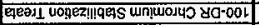 & $290950 \mathrm{~d} 38$ & 0 \\
\hline कौ है'0 & tPELO & & & & & woo lenuew uoneq & $87096-d>0$ & Oz \\
\hline Q9961 & & & & & LGS6 L & 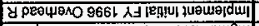 & $55096 \cdot d 08$ & 0 \\
\hline EIGZ0 & ELSZ0 & & & & & 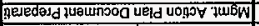 & LE095-d00 & $0: 2$ \\
\hline$\varepsilon \angle 0^{\circ} 0$ & $\varepsilon \angle 00^{\circ} 0$ & & & & & 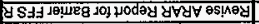 & टEOSG-d08 & 02 \\
\hline $2 \angle \angle 9^{\circ} 0$ & $2 \angle \angle 90$ & & & & & 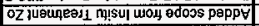 & हट0\$न के & की \\
\hline 09000 & & & & & $0500^{\circ} 0$ & 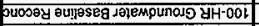 & E2095-d0日 & 02 \\
\hline 08006 & $0800 \%$ & & & & & 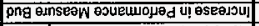 & 91096-d20 & 02 \\
\hline $0+100$ & $0+100$ & & & & & 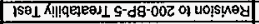 & E1096-d30 & 02 \\
\hline LOSZ 0 & 10920 & & & & & 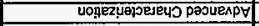 & rodo9s-d30 & क्टे \\
\hline$\angle 899^{\circ}$ & & $1 \angle 290$ & & & & 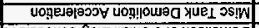 & $56196-d 30$ & 园 \\
\hline $00+1.0$ & & Optol0 & & & & 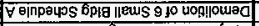 & 26196-908 & 02 \\
\hline OOEF'O & & $0051 \% 0$ & & & & 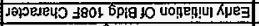 & 94-96-do日 & 02 \\
\hline OEG L'O & & $0 \varepsilon 6 \angle 0$ & & & & 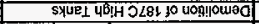 & SAl196:dOG & 02 \\
\hline $0 S \angle L^{\circ} O$ & & $0940^{\circ}$ & & & & 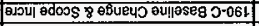 & 92195:43日 & 02 \\
\hline घहटॄ० & & $\varepsilon \varepsilon Z \varepsilon_{0}^{\circ} 0$ & & & & 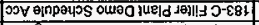 & $22+96-800$ & 02 \\
\hline $0000^{-1}$ & & $0000^{\prime}$ & & & & 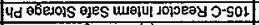 & iz/96-d00 & 02 \\
\hline $0810^{\circ} 0$ & & $0810^{\prime} 0$ & & & & 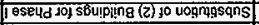 & 61196.020 & 02 \\
\hline$B \rightarrow \angle \nabla 0$ & & $t+\angle t^{\prime} 0$ & & & & 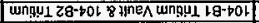 & $n+96-d 28$ & 02 \\
\hline $18497 z$ & & 18697 & & & & 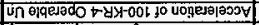 & s60036-d 08 & 0,2 \\
\hline $0000 \%$ & & 00001 & & & & 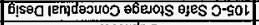 & 210056-d38 & 02 \\
\hline 00000 & & & & & & 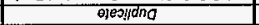 & 810908 & 을 \\
\hline$(00160)$ & & & $(005 \varepsilon 0)$ & & $\left(0099^{\circ} 0\right)$ & 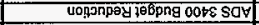 & $8 \angle 095-900$ & 02 \\
\hline$\left(208 Z^{\prime}\right)$ & & & & & (दoE'ह & 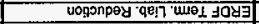 & $12000-\mathrm{d} 30$ & 02 \\
\hline$\left(7166^{\prime} 0\right)$ & & & $\left(\not+60^{\prime} 0\right)$ & & & 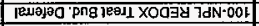 & reoso-dig & 02 \\
\hline$\left(01 s b^{\prime} 0\right)$ & & & & & (019t0) & 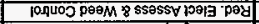 & $09006 \cdot d 00$ & 02 \\
\hline$\left(0190^{\circ} 0\right)$ & & & (01980) & & & 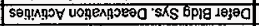 & 69056-d08 & 02 \\
\hline$(0 \leq l z-1)$ & & & (0LLE) & & & 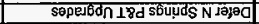 & $65005-200$ & 02 \\
\hline$(9 z 200)$ & & & & & $\left(9 z 200^{\circ} 0\right)$ & 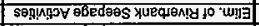 & $00096 \div 20$ & 02 \\
\hline (is900) & & & & & (ISSO0) & 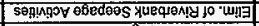 & $\operatorname{cscose-908}$ & $0 \mathrm{z}$ \\
\hline $\cos 6 z z)$ & & & $(0 \sin z)$ & & & 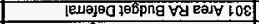 & $29095-\mathrm{d} 30$ & $0 \mathrm{z}$ \\
\hline$(10+20)$ & & & $(10950)$ & & $\left(009 k^{\circ}\right)$ & 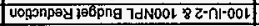 & $19036-d 90$ & 02 \\
\hline (9l0z'0) & & & & & $(910 z 0)$ & uonjonpay MO Fy & 76096-d30 & Oت? \\
\hline$(86670)$ & & & & & $866 p^{\circ} 0$ & 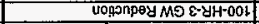 & $\varepsilon+096-\mathrm{d} 30$ & az \\
\hline$(062 l \cdot 0)$ & & & & & $(06 \angle 10)$ & 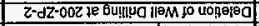 & 2 2ro96-930 & az \\
\hline$(\$ \angle P 00)$ & & & & & $\left(\nabla<+00^{\circ}\right)$ & 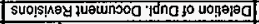 & 1+036-890 & 02 \\
\hline$(0 \mathrm{~s} s+0)$ & 00020 & & (os9tio) & & $(00<10)$ & 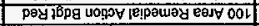 & $0+036-d 90$ & 02 \\
\hline$(0 \mathrm{~s} 180)$ & & & & & (OSIEO) & 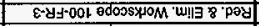 & $65096-d 30$ & $\overline{02}$ \\
\hline$(60620)$ & & & & & $\left(606 z^{\prime} 0\right)$ & 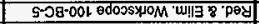 & $8 \sqrt{8006-930}$ & 02 \\
\hline$(08910)$ & & & & & $(08910)$ & 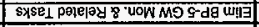 & $92006-\mathrm{d} 30$ & 02 \\
\hline $\begin{array}{l}1901 \\
9661 \\
\text { (NIS) }\end{array}$ & $\begin{array}{l}\text { swopppy } \\
\text { edoss } \\
\text { (ws) }\end{array}$ & $\begin{array}{l}\text { uopzimposory } \\
\text { odoss } \\
\text { (mst) }\end{array}$ & 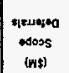 & $\begin{array}{l}\text { dojsurd1 } \\
\text { oders } \\
\text { (Wt) }\end{array}$ & $\begin{array}{l}\text { Uopessu } \\
\text { edoss } \\
\text { \{wst }\end{array}$ & 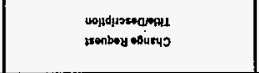 & 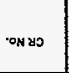 & 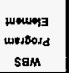 \\
\hline
\end{tabular}

1"wpa $40.80 \Lambda_{d}$

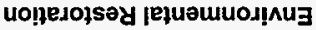

$0^{\circ} z$ 

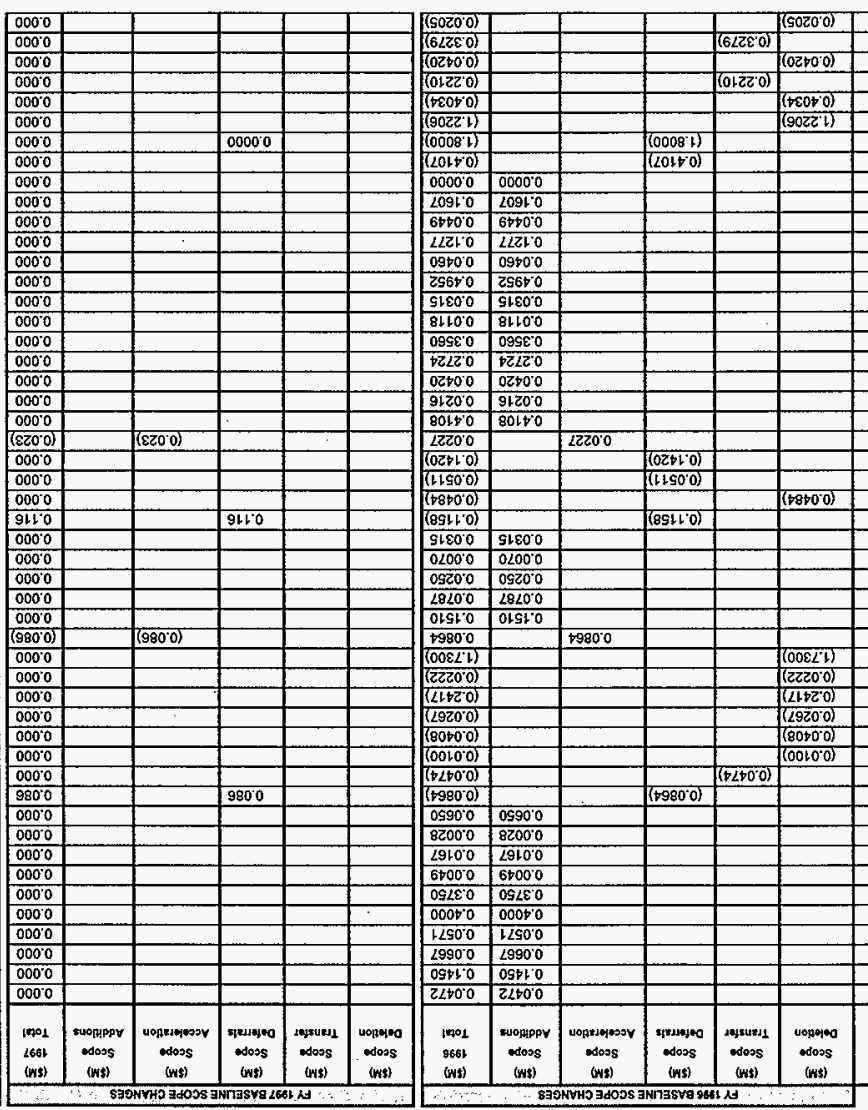

\begin{tabular}{|c|c|c|}
\hline & $01596-d \supset d$ & 02 \\
\hline & $86736-d 32$ & oz \\
\hline & $96296 \cdot d 90$ & $0 z$ \\
\hline & $46256-d 90$ & 02 \\
\hline & $88296-d 90$ & 02 \\
\hline & $69296-d 00$ & 02 \\
\hline & $23696-d 00$ & 02 \\
\hline & $\angle 6296-\mathrm{d} J 0$ & 02 \\
\hline & $80006-43 \mathrm{~d}$ & 02 \\
\hline & $60056-d 00$ & 02 \\
\hline & $80<96-d 00$ & 02 \\
\hline & $20056-\mathrm{d} 00$ & 02 \\
\hline & $80296-d 00$ & 02 \\
\hline & $\cos 96-200$ & 02 \\
\hline & $20096-d 20$ & 02 \\
\hline & $10596-d 20$ & 02 \\
\hline & $00096 \cdot d 20$ & 02 \\
\hline & $66296 \cdot d 00$ & 02 \\
\hline & 06296 -d00 & 02 \\
\hline & $\angle 8296 \mathrm{~d} 00$ & 02 \\
\hline & 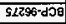 & 02 \\
\hline & $19296-d) 0$ & 02 \\
\hline & $16 z 96$-dग & oz \\
\hline & $69596-d 00$ & $0 z$ \\
\hline & Drz96-d30 & 012 \\
\hline & $09296-d>1$ & 02 \\
\hline & $58296-d 00$ & 02 \\
\hline & 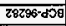 & 02 \\
\hline & $08 z 96-009$ & 02 \\
\hline & $922360 \mathrm{~d} 00$ & $\overline{02}$ \\
\hline & OEz 66 -d00 & $0 z$ \\
\hline & 66z96-dPa & 02 \\
\hline & $0,22060 \mathrm{~d} 08$ & 82 \\
\hline & r9z96-d08 & $0 z$ \\
\hline & $25 z 96 \cdot d 08$ & $\overline{07}$ \\
\hline & $\varepsilon+796.009$ & 07 \\
\hline & $27 z 86-d 08$ & 07 \\
\hline & $1+296-d \supset 8$ & 02 \\
\hline & $7 z 296 . d 08$ & 02 \\
\hline & 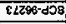 & 02 \\
\hline & $2,2256-d 08$ & 02 \\
\hline & $59256-d 38$ & 702 \\
\hline & $59296-d 08$ & 02 \\
\hline & $69296-008$ & 02 \\
\hline & $85 z 86-d 08$ & 02 \\
\hline & $25266 \cdot d 38$ & 02 \\
\hline & $55296-d 00$ & 02 \\
\hline & rszas-d08 & $0 z$ \\
\hline & 67206000 & oz \\
\hline & $97 z 96-d 00$ & $0 z$ \\
\hline \multirow{3}{*}{ 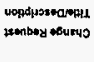 } & \multirow{3}{*}{ כצאיס } & Finverney \\
\hline & & |uFibosd \\
\hline & & $\operatorname{sen}$ \\
\hline
\end{tabular}

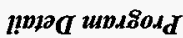

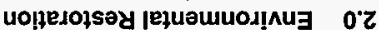

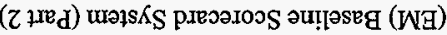


(EM) Baseline Scorecard System (Part 2)

Program Detail

\subsection{Environmental Restoration}

\begin{tabular}{|c|c|c|c|c|c|c|c|c|c|c|c|c|c|c|}
\hline & & & & & & & & & & & & & & \\
\hline & & & & FY.1 & HOBS BASELL: & ESCOPE CHA & & & & & 97 BASEU & E SCOPE CHAN & GES: & \\
\hline $\begin{array}{l}\text { Was } \\
\text { Program } \\
\text { Elomemt }\end{array}$ & CRNo. & $\begin{array}{l}\text { Change Request } \\
\text { Thtweroseription }\end{array}$ & $\begin{array}{c}\text { (SM) } \\
\text { Seope } \\
\text { Dolotion }\end{array}$ & $\begin{array}{c}\text { (sM) } \\
\text { Scope } \\
\text { Transfor }\end{array}$ & $\begin{array}{c}\text { (SM) } \\
\text { Scope } \\
\text { Dotermis }\end{array}$ & $\begin{array}{c}\text { (SM) } \\
\text { Scope } \\
\text { Acceleratton }\end{array}$ & $\begin{array}{c}\text { (SM) } \\
\text { Scope } \\
\text { Additlons }\end{array}$ & $\begin{array}{l}\text { (\$MM) } \\
\text { T9S6 } \\
\text { Total }\end{array}$ & $\begin{array}{c}\text { (\$NM) } \\
\text { Scope } \\
\text { Delotitor }\end{array}$ & $\begin{array}{c}\text { (sin) } \\
\text { Scope } \\
\text { Transtiot }\end{array}$ & $\begin{array}{c}\text { (stin) } \\
\text { Scope } \\
\text { Dofortals }\end{array}$ & $\begin{array}{c}\text { (AM) } \\
\text { Scopo } \\
\text { Accoshratton }\end{array}$ & $\begin{array}{c}\text { (SWM) } \\
\text { Scop* } \\
\text { Additions }\end{array}$ & $\begin{array}{l}\text { (SH) } \\
\text { 1397 } \\
\text { Total }\end{array}$ \\
\hline 2.0 & BCP.96311 & & $(0.0504)$ & & & & & $(0.0504)$ & & & & & & 0.000 \\
\hline 2.0 & BCP.96313 & & $(0.0200)$ & & & & & $(0.0200)$ & & & & & & 0.000 \\
\hline 2.0 & $B C P-96240$ & & & & & & 60.0500 & $(0.0500)$ & & & & & & 0.000 \\
\hline TOTAL & & & (14.7908) & (3.0631) & 5.2696 & 9.7212 & 47.9818 & 15.1187 & (1.848) & 0.000 & 10.439 & 0.026 & 1.365 & 9.983 \\
\hline
\end{tabular}

\begin{tabular}{|c|c|c|c|c|c|c|}
\hline & & TPSB BASEI & NE SCOPE CH & MGES & & \\
\hline $\begin{array}{c}\text { (SWM) } \\
\text { Scope } \\
\text { Deletion }\end{array}$ & $\begin{array}{l}\text { (SMM) } \\
\text { Scope } \\
\text { Trenster }\end{array}$ & $\begin{array}{c}\text { (SM) } \\
\text { Scopes } \\
\text { Dotertrals }\end{array}$ & $\begin{array}{c}\text { (sMi) } \\
\text { Scopt } \\
\text { Accaloration }\end{array}$ & $\begin{array}{c}\text { (SM) } \\
\text { Scope } \\
\text { Additions }\end{array}$ & $\begin{array}{l}\text { (5M) } \\
1898 \\
\text { Tolat }\end{array}$ & $\begin{array}{c}\text { (am) } \\
\text { CR } \\
\text { rorAL }\end{array}$ \\
\hline & & & & & 0.000 & $(0.050)$ \\
\hline & & & & & 0.000 & 0.020 \\
\hline & & & & & 0.000 & $(0.050)$ \\
\hline$(0.026)$ & 0.000 & 0.476 & 10.703 & 0.300 & 11.451 & 36.553 \\
\hline
\end{tabular}




\begin{tabular}{|c|c|c|c|c|c|c|}
\hline$\left(0 \varepsilon^{\circ} 0\right)$ & $00^{\circ} 0$ & $00^{\circ} 0$ & $00^{\prime} 0$ & 000 & 0000 & $00^{\circ} 0$ \\
\hline $00^{\circ} 0$ & $00^{\prime} 0$ & & & & & \\
\hline $00^{\circ} 0$ & $00^{\prime} 0$ & & & & & \\
\hline $00^{\prime} 0$ & $00^{\circ} 0$ & & & & & \\
\hline $00^{\prime} 0$ & $00^{\circ} 0$ & & & & & \\
\hline$\left(0 \varepsilon^{\circ} 0\right)$ & $00^{\circ} 0$ & & & & & \\
\hline $\begin{array}{c}7 w 101 \\
\text { 40 } \\
\text { (Ws) }\end{array}$ & $\begin{array}{l}1701 \\
8651 \\
\text { (2) }\end{array}$ & $\begin{array}{l}\text { suopppy } \\
\text { odooss } \\
\text { (Ws) }\end{array}$ & $\begin{array}{l}\text { บophumesoy } \\
\text { coos } \\
\text { (Wy) }\end{array}$ & 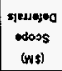 & $\begin{array}{l}\text { dostues } \\
\text { cooss } \\
\text { (ks) }\end{array}$ & $\begin{array}{l}\text { Lopono } \\
\text { edoos } \\
\text { (ks) }\end{array}$ \\
\hline
\end{tabular}

\begin{tabular}{|c|c|c|c|c|c|}
\hline $00^{\circ} 0$ & $100^{\circ} 0$ & 000 & $100^{\circ} 0$ & $00^{\prime} 0$ & 000 \\
\hline \multicolumn{6}{|l|}{$00^{\circ} 0$} \\
\hline \multirow{2}{*}{\multicolumn{6}{|c|}{$\begin{array}{l}00^{\circ} 0 \\
00^{\circ} 0\end{array}$}} \\
\hline & & & & & \\
\hline \multicolumn{6}{|l|}{$00^{\circ} 0$} \\
\hline \multicolumn{6}{|l|}{$00 \%$} \\
\hline $\begin{array}{l}1701 \\
2681 \\
\text { (W(s) }\end{array}$ & $\begin{array}{l}\text { suorhppy } \\
\text { edoss } \\
\text { CW\$Y }\end{array}$ & $\begin{array}{c}\text { Hopesmesosy } \\
\text { cososs } \\
\text { (Mt) }\end{array}$ & 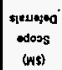 & $\begin{array}{l}\text { noysues } 1 \\
\text { odoos } \\
\text { (inst) }\end{array}$ & $\begin{array}{l}\text { uosenos } \\
\text { onsss) } \\
\text { (wss) }\end{array}$ \\
\hline
\end{tabular}

\begin{tabular}{|c|c|c|c|c|c|c|c|c|}
\hline$\left(0 \varepsilon^{\circ} 0\right)$ & $00^{\circ} 0$ & 000 & $00^{\circ} 0$ & 000 & $\left(0 \varepsilon^{\circ} 0\right)$ & & & 70101 \\
\hline $00^{\circ}$ & & & & & & & & \\
\hline 000 & & & & & & & & \\
\hline 000 & & & & & & & & \\
\hline 000 & & & & & & & & \\
\hline$\left(0 \varepsilon_{0}\right)$ & & & & & $\left(0 \varepsilon^{\prime} 0\right)$ & 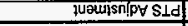 & & \\
\hline $\begin{array}{l}1001 \\
9601 \\
\text { (Ws) }\end{array}$ & $\begin{array}{l}\text { * YORppy } \\
\text { cooss } \\
\text { (Ws) }\end{array}$ & $\begin{array}{l}\text { Hoperyoss } \\
\text { cocoss } \\
\text { (ws) }\end{array}$ & 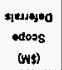 & $\begin{array}{l}\text { دeysurul } \\
\text { odoss } \\
\text { (W:s) }\end{array}$ & $\begin{array}{l}\text { wopined } \\
\text { ecoss } \\
\text { [Ws] }\end{array}$ & 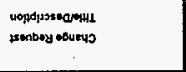 & •N & $\begin{array}{l}\text { มूowely } \\
\text { weisosd } \\
\text { SgM }\end{array}$ \\
\hline
\end{tabular}

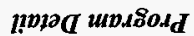

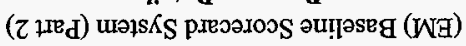

1+26-7y 


\begin{tabular}{|c|c|c|c|c|c|c|}
\hline (S98'z) & $\left(16 L^{\prime} \varepsilon\right]$ & $000 \% 0$ & $000^{\prime} 0$ & $000^{\prime} 0$ & $000^{\circ} 0$ & $\left(\mathcal{L} B L^{\prime} \varepsilon\right)$ \\
\hline $7 \angle L^{\prime} 0$ & $000^{\circ} 0$ & & & & & \\
\hline $889^{\circ} 0$ & $000^{\circ} 0$ & & & & & \\
\hline $598^{\circ} 0$ & $000^{\circ} 0$ & & & & & \\
\hline$\angle 66^{\circ} \mathrm{I}$ & $000^{\circ} 0$ & & & & & \\
\hline 0000 & $000^{\circ} 0$ & & & & & \\
\hline $000^{\circ} 0$ & 0000 & & & & & \\
\hline $68 \varepsilon^{\circ} 0$ & 0000 & & & & & \\
\hline$\left(z z s^{\prime} 9\right)$ & $000^{\circ} 0$ & & & & & \\
\hline$\left(009^{\circ} 0\right)$ & 0000 & & & & & \\
\hline $56 Z 0$ & $000^{\circ} 0$ & & & & & \\
\hline (16LE) & $(16 L \bar{l})$ & & & & & $\left(16 L^{\prime} \varepsilon\right)$ \\
\hline $9 \angle \angle 00$ & 0000 & & & & & \\
\hline slto 0 & $000^{\circ} 0$ & & & & & \\
\hline $508 Z$ & 0000 & & & & & \\
\hline 0000 & 0000 & & & & & \\
\hline $\begin{array}{l}\text { w101 } \\
\text { yo } \\
\text { (W:s) }\end{array}$ & $\begin{array}{l}1001 \\
8661 \\
(w 5)\end{array}$ & $\begin{array}{l}\text { tuopppy } \\
\text { edoos } \\
\text { (W:) }\end{array}$ & 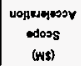 & $\begin{array}{l}\text { Niruejea } \\
\text { cooss } \\
\text { (wis) }\end{array}$ & $\begin{array}{l}\text { doysurst } \\
\text { cooss } \\
\text { (W:s) }\end{array}$ & $\begin{array}{l}\text { wophen } \\
\text { ocoss } \\
\text { (N) } 5\}\end{array}$ \\
\hline
\end{tabular}

\begin{tabular}{|c|c|c|c|c|c|}
\hline LLL'Z & $69 Z^{\prime \prime} 0$ & $000^{\circ} 0$ & $2+8 \cdot 1$ & EZE"Z & (£टع"Z) \\
\hline $296^{\circ} 0$ & & & $296^{\circ} 0$ & & \\
\hline $69 Z^{\prime} 0$ & $692^{\prime} 0$ & & & & \\
\hline $988^{\prime} 0$ & & & $998^{\circ} 0$ & & \\
\hline \multicolumn{6}{|l|}{$000^{\circ} 0$} \\
\hline slo"o & & & S10'0 & & \\
\hline \multicolumn{6}{|l|}{$000^{\circ} 0$} \\
\hline \multicolumn{6}{|l|}{$000^{\circ} 0$} \\
\hline \multicolumn{6}{|l|}{0000} \\
\hline \multicolumn{6}{|l|}{0000} \\
\hline \multicolumn{6}{|l|}{$000^{\circ} 0$} \\
\hline \multicolumn{6}{|l|}{0000} \\
\hline \multicolumn{6}{|l|}{0000} \\
\hline \multirow{2}{*}{\multicolumn{6}{|c|}{$\begin{array}{l}000^{\circ} 0 \\
000^{\circ} 0\end{array}$}} \\
\hline & & & & & \\
\hline \multicolumn{4}{|l|}{0000} & \begin{tabular}{|l|}
$\varepsilon Z \varepsilon z$ \\
\end{tabular} & (दzєz) \\
\hline $\begin{array}{l}\text { lpos } \\
\text { 26s! } \\
\text { (WSS) }\end{array}$ & $\begin{array}{l}\text { suopippy } \\
\text { ocoss } \\
\text { (Wf\}) }\end{array}$ & $\begin{array}{c}\text { uoperiposy } \\
\text { cdoss } \\
\text { (is) }\end{array}$ & 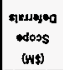 & $\begin{array}{l}\text { 19ysund } \\
\text { cocoss } \\
\text { (WWt) }\end{array}$ & $\begin{array}{l}\text { uonmwo } \\
\text { moss } \\
\text { (kis) }\end{array}$ \\
\hline & & VHO $3400 S$ & NhaSVY $\angle$ & $\lambda$ ? & \\
\hline
\end{tabular}

\begin{tabular}{|c|c|c|c|c|c|c|c|c|}
\hline$\left(581^{-12)}\right.$ & BEEZ & $00 Z^{\prime \prime}$ & $10 \angle 0$ & (268'0) & (£\&O's) & & & $7 \forall 101$ \\
\hline$\left(881^{\circ} 0\right)$ & & 0021 & $(2960)$ & & $\left(92 b^{\prime} 0\right)$ & & $300-96-d d$ & \\
\hline tIE & $889^{\circ} 0$ & & $(6980)$ & & $000^{\circ} 0$ & & sto-s6-d1 & \\
\hline $000^{\circ} 0$ & $\varepsilon ट L^{\prime}$ & & $\left(998^{\circ} 0\right)$ & $880^{\circ} 0$ & $\left(9 \circ \varepsilon^{\circ} 0\right)$ & & $9200-86-\mathrm{d1}$ & \\
\hline$\angle 66^{\circ} \mathrm{I}$ & & & $\angle 66^{\circ}$ & & & & $6100 \cdot 66 \cdot \mathrm{d1}$ & \\
\hline (sion) & & & $\left(\mathrm{Gl} 0^{\circ}\right)$ & & & & $2200-86 \cdot \mathrm{dL}$ & \\
\hline $000^{\prime} 0$ & & & $000^{\circ} 0$ & $020^{\circ} 0$ & $\left(020^{\circ} 0\right)$ & & $6200-96 \cdot d 1$ & \\
\hline $68 \varepsilon^{\prime} 0$ & $68 \varepsilon^{\circ} 0$ & & & & & & $z 200-66 \cdot d 1$ & \\
\hline (zZg'9) & & & (96є'Z) & $(88 z " 2)$ & $\left(888^{\circ}\right)$ & & $240-96 \cdot \mathrm{d1}$ & \\
\hline$\left(00 g^{\prime} 0\right)$ & & & & $\left(00 g^{\circ} 0\right)$ & & & $710-96 \cdot d 1$ & \\
\hline$\$ 6 Z 0$ & $t \quad Z=0$ & & & & & & 14900-96-d1 & \\
\hline $000^{\circ} 0$ & $000^{\prime} 0$ & $000^{\circ} 0$ & $000^{\circ} 0$ & $000^{\prime} 0$ & $000^{\prime} 0$ & & $200-86-d 1$ & \\
\hline $9 \angle E 0$ & & & $9 \angle \varepsilon^{\prime} 0$ & & & & ob 50 서 & \\
\hline SlDO & & & $0 E s 0$ & & (SLI'O) & & OrO 56 서 & \\
\hline $\operatorname{soc} 2$ & & & SOE'? & & & & OD $50 \mathrm{ASA}$ & \\
\hline 0000 & & & & $88 Z Z$ & $(88 Z 7)$ & 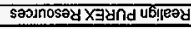 & $010-86 \mathrm{dl}$ & 12 \\
\hline $\begin{array}{l}1 \text { 1901 } \\
966 t \\
\text { (Hs) }\end{array}$ & $\begin{array}{l}\text { suopppy } \\
\text { octoss } \\
\text { (H:S) }\end{array}$ & 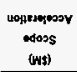 & $\begin{array}{l}\text { sาumed } \\
\text { ocoss } \\
\text { (HSs) }\end{array}$ & 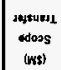 & $\begin{array}{l}\text { морена } \\
\text { esors } \\
\text { (ws) }\end{array}$ & 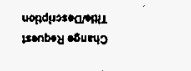 & ON & 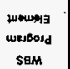 \\
\hline
\end{tabular}

\section{limpa un.180 d}

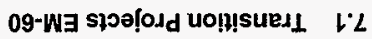

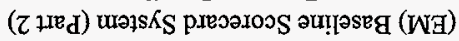




\begin{tabular}{|c|c|c|c|c|c|c|}
\hline LLLZ & $\left(000^{2} 2\right)$ & $000^{\circ} 0$ & $\left(000^{\circ} 2\right)$ & 0000 & $000^{\circ} 0$ & $000^{\circ} 0$ \\
\hline $0{ }^{0} g^{\prime} 0$ & $000^{\circ} 0$ & & & & & \\
\hline $000^{\circ} 0$ & $000^{\circ} 0$ & & & & & \\
\hline 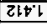 & $000^{\circ} 0$ & & & & & \\
\hline 090 & $000^{\circ}$ & & & & & \\
\hline $000^{\prime} 0$ & $000^{\circ} 0$ & & & & & \\
\hline SOL'0 & 0000 & & & & & \\
\hline $000^{\circ} 0$ & $\left(00^{\circ} \mathrm{Z}\right)$ & & $\left(000^{\circ} \mathrm{Z}\right)$ & & & \\
\hline $000^{\circ} 0$ & $000^{\circ} 0$ & & & & & \\
\hline $000^{\prime} 0$ & $000^{\circ} 0$ & & & & & \\
\hline $000^{\prime} 0$ & $000^{\prime} 0$ & & & & & \\
\hline $\begin{array}{c}\text { W101 } \\
\text { \&D } \\
\text { (ws) }\end{array}$ & $\begin{array}{l}1001 \\
9851 \\
\text { (W:5) }\end{array}$ & $\begin{array}{l}\text { suppppy } \\
\text { edoss } \\
\text { (Ws) }\end{array}$ & $\begin{array}{c}\text { uoperesery } \\
\text { edoss } \\
\text { (Ws) }\end{array}$ & 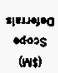 & $\begin{array}{l}\text { sogrues } \\
\text { octoss } \\
\text { (Ks) }\end{array}$ & $\begin{array}{l}\text { uophen } \\
\text { odors } \\
\text { [N:s] }\end{array}$ \\
\hline
\end{tabular}

\begin{tabular}{|c|c|c|c|c|c|}
\hline (OLI'L) & $000^{\prime} 0$ & $\left(668^{\prime} \mathrm{t}\right)$ & $68 Z^{\prime} 0$ & $000^{\circ} 0$ & $000^{\prime} 0$ \\
\hline \\
\hline$\left(000^{\circ}\right)$ & & $(0080)$ & & & \\
\hline \multicolumn{6}{|l|}{$000^{\circ} 0$} \\
\hline \multicolumn{6}{|l|}{0000} \\
\hline $0+0^{\circ} 0$ & & & 0000 & & \\
\hline $000^{\circ} 0$ & & & & & \\
\hline $005^{\prime} t$ & & 0091 & & & \\
\hline$\left(00 t^{\prime} 0\right)$ & & $\left(000^{\circ} 0\right)$ & & & \\
\hline $6+Z 70$ & & & 6620 & & \\
\hline$\left(669^{\prime} z\right)$ & & $(6697)$ & & & \\
\hline $\begin{array}{l}1701 \\
2661 \\
\text { (N) }\end{array}$ & 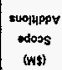 & $\begin{array}{c}\text { wongeroposy } \\
\text { idess } \\
\text { (KS) }\end{array}$ & 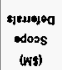 & $\begin{array}{c}\text { Jojsues } \\
\text { edoos } \\
\text { (4月, }\end{array}$ & $\begin{array}{l}\text { voppene } \\
\text { ocoss } \\
\text { (WAs) }\end{array}$ \\
\hline
\end{tabular}

\begin{tabular}{|c|c|c|c|c|c|c|c|c|}
\hline$Z Z L \mathrm{~s}$ & LLLZZ & $668^{\circ} \varepsilon$ & $(68 \mathrm{CO})$ & $000^{\circ} 0$ & $000^{\prime} 0$ & & & $7 \forall 101$ \\
\hline OSg'0 & 0 og'o & & & & & 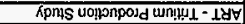 & $560-96-1 d y$ & \\
\hline $00 \varepsilon^{\prime} 0$ & & 0080 & & & & 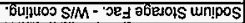 & 6LO-96-18YY & \\
\hline Z1F' & ZIt't & & & & & Sa6ueyo SIM Lلy & $2<0-96-1 \mathrm{lyV}$ & \\
\hline $050^{\prime} 0$ & $050^{\circ} 0$ & & & & & 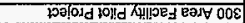 & $850-96: 14 \bar{y}$ & \\
\hline$(0+0)$ & & & $(0+00)$ & & & $10 Z-96-618$ S/N 10 d duos daja & $850-96-1 \mathrm{dV}$ & \\
\hline 5010 & 5010 & & & & & 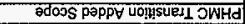 & $090-56 \cdot 18 y$ & IE2 \\
\hline $005^{\prime} 0$ & & 0050 & & & & 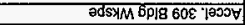 & $000-96-194$ & $\overline{\varepsilon^{2}}$ \\
\hline 000 & & 000 & & & & 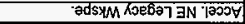 & $200-96-18 y$ & $\sqrt{182}$ \\
\hline$(6+20)$ & & & $(6 \nabla Z 70)$ & & & 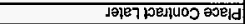 & $520-56 \div-18 y$ & $\overline{E L}$ \\
\hline $669 \mathrm{z}$ & & $669^{\circ} \mathrm{Z}$ & & & & 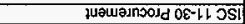 & $0\{0-963-14 y$ & IE \\
\hline $\begin{array}{l}\text { Inol } \\
968 \mathrm{~b} \\
(\mathrm{~W} 9)\end{array}$ & $\begin{array}{l}\text { ELOPIPPY } \\
\text { odoss } \\
\text { (WS) }\end{array}$ & 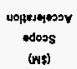 & $\begin{array}{l}\text { squwano } \\
\text { cooss } \\
\text { (ws) }\end{array}$ & $\begin{array}{l}\text { mejsuest } \\
\text { odoos } \\
\text { (WIS) }\end{array}$ & $\begin{array}{l}\text { Uopenos } \\
\text { odoss } \\
\text { (1H:) }\end{array}$ & 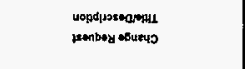 & כערים & 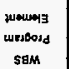 \\
\hline
\end{tabular}

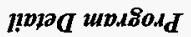

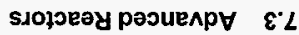

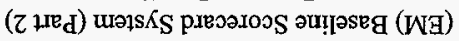




\begin{tabular}{|c|c|c|c|c|c|c|}
\hline $00^{\circ} 0$ & $00^{\circ} 0$ & $00^{\circ} 0$ & $00^{\circ} 0$ & 000 & $00^{\prime} 0$ & $00^{\circ} 0$ \\
\hline 000 & $00^{\circ} 0$ & & & & & \\
\hline $00^{\circ} 0$ & $00^{\circ} 0$ & & & & & \\
\hline $00^{\circ} 0$ & $00^{\circ} 0$ & & & & & \\
\hline $00^{\circ} 0$ & \begin{tabular}{|l|l}
$00^{\circ} 0$ \\
\end{tabular} & & & & & \\
\hline $00^{\circ} 0$ & $00^{\circ} 0$ & & & & & \\
\hline $\begin{array}{c}\text { 7vol } \\
\text { yo } \\
\text { (WH3 }\end{array}$ & $\begin{array}{l}1001 \\
8603 \\
\text { (wis) }\end{array}$ & $\begin{array}{l}\text { shopppy } \\
\text { edoss } \\
\text { (ws) }\end{array}$ & 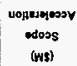 & 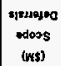 & 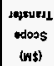 & $\begin{array}{l}\text { uopiwe } \\
\text { odoss } \\
\text { (Ws) }\end{array}$ \\
\hline
\end{tabular}

\begin{tabular}{|c|c|c|c|c|c|}
\hline $00^{\circ} 0$ & 000 & $00^{\circ} 0$ & $00^{\circ} 0$ & $00^{\circ} 0$ & 000 \\
\hline 000 & & & & & \\
\hline \multicolumn{6}{|l|}{$00^{\prime} 0$} \\
\hline \multicolumn{6}{|l|}{$00^{\circ} 0$} \\
\hline \multirow{2}{*}{\multicolumn{6}{|c|}{$\frac{00^{\circ} 0}{00^{\prime} 0}$}} \\
\hline & & & & & \\
\hline $\begin{array}{l}1701 \\
2661 \\
(1 \times 1)\end{array}$ & $\begin{array}{l}\text { suojyppr } \\
\text { soloss } \\
\text { (सHs) }\end{array}$ & $\begin{array}{l}\text { uopereyesor } \\
\text { edoss } \\
\text { (ws) }\end{array}$ & $\begin{array}{l}\text { speseyng } \\
\text { edoxs } \\
\text { (ws) }\end{array}$ & $\begin{array}{l}\text { sojsurss } \\
\text { edors } \\
\text { (ws) }\end{array}$ & 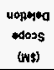 \\
\hline
\end{tabular}

\begin{tabular}{|c|c|c|c|c|c|c|c|c|}
\hline 000 & $00^{\circ} 0$ & 000 & 000 & $00^{\circ} 0$ & $00^{\circ} 0$ & & & $7 \forall 101$ \\
\hline \multicolumn{9}{|l|}{000} \\
\hline \multirow{2}{*}{\multicolumn{9}{|c|}{$\frac{00^{\circ} 0}{00^{\prime} 0}$}} \\
\hline & & & & & & & & \\
\hline \multicolumn{9}{|l|}{$00^{\prime} 0$} \\
\hline $00^{\circ} 0$ & & & & & & & & \\
\hline $\begin{array}{l}1401 \\
9681 \\
(415)\end{array}$ & 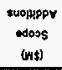 & $\begin{array}{l}\text { woperuesory } \\
\text { edors } \\
\text { (w19) }\end{array}$ & 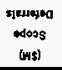 & 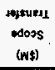 & 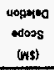 & 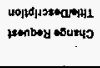 & סN & 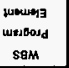 \\
\hline
\end{tabular}

\section{1!moa und8odd}

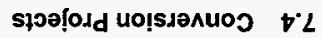

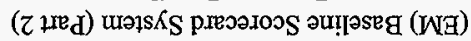




\begin{tabular}{|c|c|c|c|c|c|}
\hline $00^{\circ} 0$ & $60^{\circ} 0$ & $00^{\prime} 0$ & 000 & $00^{\circ} 0$ & $00^{\prime} 0$ \\
\hline \multicolumn{6}{|l|}{000} \\
\hline \multicolumn{6}{|l|}{000} \\
\hline \multicolumn{6}{|l|}{000} \\
\hline \multirow{2}{*}{\multicolumn{6}{|c|}{$\frac{00^{\circ} 0}{00^{\circ} 0}$}} \\
\hline & & & & & \\
\hline $\begin{array}{l}1001 \\
2665 \\
(1215)\end{array}$ & $\begin{array}{l}\text { suopppy } \\
\text { odess } \\
\text { (Wis) }\end{array}$ & $\begin{array}{l}\text { uopespoosy } \\
\text { wors } \\
\text { (w:) }\end{array}$ & 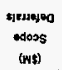 & $\begin{array}{l}\text { sejsutei1 } \\
\text { coloss } \\
\text { (Wy) }\end{array}$ & $\begin{array}{l}\text { черн*0 } \\
\text { *doss } \\
\text { (Ws) }\end{array}$ \\
\hline & & WD & nasve $\angle 6$ & & \\
\hline
\end{tabular}

\begin{tabular}{|c|c|c|c|c|c|c|c|c|}
\hline $0 \varepsilon^{\prime \prime}$ & $08 \%$ & 000 & $00^{\circ} 0$ & 000 & $00^{\circ} 0$ & & & $7 \forall 101$ \\
\hline 000 & & & & & & & & \\
\hline 000 & & & & & & & & \\
\hline $00^{\circ} 0$ & & & & & & & & \\
\hline 000 & & & & & & & & \\
\hline $0 \varepsilon^{\prime} b$ & $0 \varepsilon^{\prime} t$ & & & & & uognsuej $\perp$ OWHd & & \\
\hline $\begin{array}{l}1402 \\
9681 \\
(145)\end{array}$ & $\begin{array}{l}\text { suoppppy } \\
\text { edors } \\
\text { (W:s) }\end{array}$ & 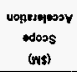 & 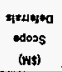 & $\begin{array}{l}\text { Iosures } \\
\text { Nooss } \\
\text { (ws) }\end{array}$ & $\begin{array}{l}\text { uolinen } \\
\text { ososs } \\
\text { (sis) }\end{array}$ & 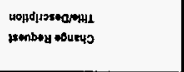 & ON & $\begin{array}{l}\text { moundey } \\
\text { wrisosd } \\
\text { Sam }\end{array}$ \\
\hline
\end{tabular}

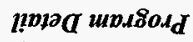




\begin{tabular}{|c|c|c|c|c|c|c|}
\hline (LEF'L) & $0 s z_{0}$ & $000^{\circ} 0$ & 0000 & OSE 0 & $000^{\circ} 0$ & 0000 \\
\hline $000^{\circ} 0$ & 0000 & & & & & \\
\hline $000^{\circ} 0$ & 0000 & & & & & \\
\hline $000^{\circ} 0$ & 0000 & & & & & \\
\hline$(t \in L Z)$ & \begin{tabular}{|c|c|}
$000^{\circ}$ \\
\end{tabular} & & & & & \\
\hline $000^{\circ} 0$ & 0000 & & . & & & \\
\hline$\left(625^{\circ} 0\right)$ & 0000 & & & & & \\
\hline 0600 & 0000 & & & & & \\
\hline 0000 & 0000 & & & & & \\
\hline$\left(089^{\prime} \phi\right)$ & OSZ'0 & & & Osz'0 & & \\
\hline$(\overline{8 \varepsilon}+0)$ & $000^{\circ} 0$ & & & & & \\
\hline 0060 & 0000 & & & & & \\
\hline $\begin{array}{c}\text { w1or } \\
\text { yo } \\
\text { (H:s) }\end{array}$ & $\begin{array}{l}1901 \\
5661 \\
(469)\end{array}$ & $\begin{array}{l}\text { Suoptpoy } \\
\text { edoss } \\
\text { (ws) }\end{array}$ & $\begin{array}{l}\text { Uopergoss } \\
\text { adoss } \\
\text { (WS) }\end{array}$ & $\begin{array}{l}\text { sitcused } \\
\text { odors } \\
\text { (N)\$) }\end{array}$ & $\begin{array}{l}\text { dessures } \\
\text { edoss } \\
\text { (Wts) }\end{array}$ & $\begin{array}{l}\text { Uopm*d } \\
\text { ocloos } \\
\text { (Nis) }\end{array}$ \\
\hline & 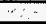 & $\therefore 998$ & nojdo0s & n̄sve of & & \\
\hline
\end{tabular}

\begin{tabular}{|c|c|c|c|c|c|}
\hline $69 L L$ & $000^{\circ} 0$ & $\left(689^{\prime} 0\right)$ & $800^{\circ} \mathrm{E}$ & $000^{\circ} 0$ & $\left(00 L^{\prime} 0\right)$ \\
\hline \multicolumn{6}{|l|}{$000^{\circ} 0$} \\
\hline \multicolumn{6}{|l|}{0000} \\
\hline \multicolumn{6}{|l|}{0000} \\
\hline \multicolumn{6}{|l|}{0000} \\
\hline \multicolumn{6}{|l|}{0000} \\
\hline$(6 \mathrm{zg} 0)$ & & $\left(6 z 9^{\circ} 0\right)$ & & & \\
\hline 0600 & & $(0100)$ & $00 r^{\circ} 0$ & & \\
\hline \multicolumn{6}{|l|}{$000^{\circ} 0$} \\
\hline $80 z \bar{Z}$ & & & 8062 & & $(00<0)$ \\
\hline \multicolumn{6}{|l|}{$000^{\circ} 0$} \\
\hline \multicolumn{6}{|l|}{0000} \\
\hline $\begin{array}{l}1001 \\
2681 \\
\text { (ks) }\end{array}$ & $\begin{array}{l}\text { suopppy } \\
\text { моors } \\
\text { (w:) }\end{array}$ & $\begin{array}{l}\text { coperyososy } \\
\text { noss } \\
\text { (its) }\end{array}$ & 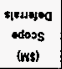 & 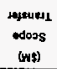 & 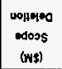 \\
\hline-1 & & VH0.adojs: & $73579 \angle 6$ & A & \\
\hline
\end{tabular}

\begin{tabular}{|c|c|c|c|c|c|c|c|c|}
\hline$\left(0 \mathrm{Sp}^{*} 6\right)$ & $\angle t S \varepsilon$ & $689^{\circ} 0$ & (9Lع') & 0000 & [(0zZ0L) & & & $7 \forall \perp O 1$ \\
\hline \multirow{2}{*}{\multicolumn{9}{|c|}{\begin{tabular}{|l|}
0000 \\
00
\end{tabular}}} \\
\hline $000^{\prime} 0$ & & & & & & & & \\
\hline \multicolumn{9}{|l|}{$000^{\circ} 0$} \\
\hline$(7 \angle L Z)$ & $000^{\circ} 0$ & 0000 & 0000 & 0000 & $(\bar{\sigma} L L C)$ & & CSO $96 \mathrm{KAO}$ & \\
\hline $000^{\circ} 0$ & $\angle \angle S O$ & 0000 & 0000 & $000^{\circ} 0$ & $\left(\angle L \mathcal{G}^{\circ} 0\right)$ & & Sh0-36-kd & \\
\hline 0000 & 0000 & $6 z \mathrm{SO}^{\circ}$ & $000^{\circ} 0$ & 0000 & $\left(629^{\circ} 0\right)$ & & $250-96-\mathrm{Wd}$ & \\
\hline$\left(000^{\circ}\right)$ & $060^{\circ} 0$ & 0100 & $\left(000^{\circ}\right)$ & $000^{\circ} 0$ & $000^{\circ} 0$ & & $620-96 \%$ Wd & \\
\hline 0000 & OSZ' & $000^{\circ}$ & $000^{\circ} 0$ & $000^{\circ} 0$ & $(\log 21)$ & & $800-96-W d 7$ & \\
\hline$(8 \varepsilon+2)$ & $089^{\circ} \mathrm{L}$ & $000^{\circ} 0$ & $\left(8 \angle 99^{\circ} \varepsilon\right)$ & $000^{\circ} 0$ & $\left(060^{\circ} \mathrm{G}\right)$ & & $820-96$ wd & \\
\hline$(8 \varepsilon+0)$ & $000^{\circ} 0$ & 0000 & $\left(8 E b^{\prime} 0\right)$ & $000^{\circ} 0$ & $000^{\circ} 0$ & 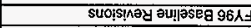 & $010-96 \cdot \mathrm{WJ}$ & \\
\hline $006^{\prime} 0$ & & & 0060 & & & & OCDS64 & 0005 \\
\hline $\begin{array}{l}1401 \\
9661 \\
(W \$ 5)\end{array}$ & $\begin{array}{l}\text { *uequppY } \\
\text { odoos } \\
\text { (Wws) }\end{array}$ & $\begin{array}{c}\text { copecoesosor } \\
\text { edoos } \\
\text { (wss) }\end{array}$ & 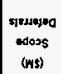 & 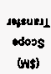 & $\begin{array}{l}\text { एopiq日a } \\
\text { odors } \\
\text { (WF) }\end{array}$ & 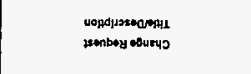 & פי צ' & 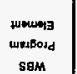 \\
\hline
\end{tabular}

\section{1!ploa updsoid}

pมорuвา S'L

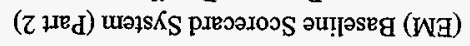

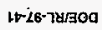

